ARTICLE

https://doi.org/10.1038/s41467-019-08875-x

\title{
Persistent DNA-break potential near telomeres increases initiation of meiotic recombination on short chromosomes
}

\author{
Vijayalakshmi V. Subramanian (10) ', Xuan Zhu (10) 2,6, Tovah E. Markowitz ${ }^{1,7}$, Luis A. Vale-Silva (i) ${ }^{1,8}$, \\ Pedro A. San-Segundo (1) ${ }^{3}$, Nancy M. Hollingsworth (1) ${ }^{4}$, Scott Keeney (1) ${ }^{2,5}$ \& Andreas Hochwagen (1) ${ }^{1}$
}

Faithful meiotic chromosome inheritance and fertility rely on the stimulation of meiotic crossover recombination by potentially genotoxic DNA double-strand breaks (DSBs). To avoid excessive damage, feedback mechanisms down-regulate DSBs, likely in response to initiation of crossover repair. In Saccharomyces cerevisiae, this regulation requires the removal of the conserved DSB-promoting protein Hop1/HORMAD during chromosome synapsis. Here, we identify privileged end-adjacent regions (EARs) spanning roughly $100 \mathrm{~kb}$ near all telomeres that escape DSB down-regulation. These regions retain Hop1 and continue to break in pachynema despite normal synaptonemal complex deposition. Differential retention of Hop1 requires the disassemblase Pch2/TRIP13, which preferentially removes Hop1 from telomere-distant sequences, and is modulated by the histone deacetylase Sir2 and the nucleoporin Nup2. Importantly, the uniform size of EARs among chromosomes contributes to disproportionately high DSB and repair signals on short chromosomes in pachynema, suggesting that EARs partially underlie the curiously high recombination rate of short chromosomes.

\footnotetext{
${ }^{1}$ Department of Biology, New York University, New York, NY 10003, USA. ${ }^{2}$ Molecular Biology Program, Memorial Sloan Kettering Cancer Center, New York, NY 10065, USA. ${ }^{3}$ Instituto de Biología Funcional y Genómica, Consejo Superior de Investigaciones Científicas, University of Salamanca, 37007 Salamanca, Spain. ${ }^{4}$ Department of Biochemistry and Cell Biology, Stony Brook University, Stony Brook, NY 11794, USA. ${ }^{5}$ Howard Hughes Medical Institute, Memorial Sloan Kettering Cancer Center, New York, NY 10065, USA. ${ }^{6}$ Present address: Amazon Al, Seattle, WA 98101, USA. 'Present address: Frederick National Laboratory for Cancer Research, Frederick, MD 21701, USA. ${ }^{8}$ Present address: BioQuant Center, Heidelberg University, 69120 Heidelberg, Germany.

Correspondence and requests for materials should be addressed to A.H. (email: andi@nyu.edu)
} 
$\mathrm{M}$ eiosis generates haploid sex cells using two consecutive chromosome segregation events that follow a single cycle of DNA replication. To assist proper separation of homologous chromosomes in the first segregation phase (meiosis I), numerous DNA double-strand breaks (DSBs) are introduced by topoisomerase-like enzyme Spo11 to stimulate the formation of crossover recombination products (COs). Together with sister chromatid cohesion, COs connect homologous chromosome pairs and promote their correct alignment on the meiosis I spindle ${ }^{1,2}$

Because DSBs are potentially genotoxic, several processes choreograph DSB formation at the right place and time to maintain genome integrity ${ }^{1-4}$. DSBs form preferentially at hotspots that depend strongly on chromatin accessibility and appropriate histone modifications ${ }^{1,5}$. In addition, Spo11 activity is modulated over larger chromosomal domains by specialized chromosome architecture, in which chromatin loops emanate from a meiosis-specific protein axis (the axial element). In this architecture, DSB hotspots are primarily found on chromatin loops but are thought to translocate to the axial element to encounter proteins necessary for DSB formation ${ }^{6,7}$. Accordingly, mutants lacking axial-element proteins exhibit severely reduced DSB levels ${ }^{8,9}$. The enrichment profile of axial-element proteins correlates well with DSB levels on a broad, regional scale ${ }^{6,7,10}$, suggesting that controlled distribution of these proteins is important for governing the distribution of meiotic DSB activity.

In addition, a network of checkpoint and feedback mechanisms controls the timing of DSB formation ${ }^{2-5}$. These mechanisms establish a defined window of opportunity for DSB formation by preventing DSB formation during pre-meiotic DNA replication, as well as upon exit from meiotic prophase ${ }^{11-17}$. Checkpoint mechanisms also suppress redundant DSB formation in the vicinity of already broken $\mathrm{DNA}^{18-21}$. In addition, DSBs are progressively down-regulated as prophase proceeds. Studies suggest that the synaptonemal complex (SC), an evolutionarily conserved proteinaceous structure that assembles between homologous chromosomes, is responsible for this process $16,22-24$. The SC is thought to ensure cessation of DSB formation in a chromosome-autonomous fashion and likely triggers DSB downregulation following initiation of the obligatory $\mathrm{CO}$ on a given chromosome pair ${ }^{16,22-26}$.

In S. cerevisiae, SC-dependent down-regulation of DSB activity is linked to the chromosomal reduction of the axis-associated HORMA-domain protein Hop1, which normally recruits DNA break machinery to the meiotic chromatin ${ }^{7,23}$. Reduction of chromosomal Hop1 coincides with SC assembly and depends on SC-mediated recruitment of the $\mathrm{AAA}^{+}$-ATPase Pch2 $23,27-29$. In the absence of $\mathrm{PCH} 2$, Hop 1 signal continues to accumulate on synapsed chromosomes. A similar process is observed in mouse spermatocytes ${ }^{24,30}$. Intriguingly, not all DSB hotspots in yeast are equally dampened upon SC assembly. A number of hotspots, including widely used model hotspots (e.g., YCR047C and HIS4LEU2-a modified hotspot at YCL030C), remain competent for DSB formation throughout prophase, irrespective of the presence of the $S^{11,23,31}$. The origin and purpose of these longlived hotspots is unknown.

One possible function of long-lived hotspots is to increase the window of opportunity for DSB formation on short chromosomes. Short chromosomes exhibit elevated recombination density in many organisms ${ }^{16,32-34}$. In yeast, this bias is already apparent during DSB formation ${ }^{35-38}$ and is likely driven by two distinct mechanisms, both of which remain poorly understood. The first mechanism enriches axis proteins and DSB factors on short chromosomes and is independent of DSB formation ${ }^{7,10}$. The second mechanism is thought to involve the SC-dependent down-regulation of DSBs linked to homologue engagement for
DSB repair ${ }^{16}$. It has been proposed that shorter chromosomes are slower at engaging with their homologue, leading to prolonged DSB activity specifically on these chromosomes ${ }^{16}$. Accordingly, general disruption of homologue engagement leads to continued DSB formation on all chromosomes and a loss of DSB enrichment on short chromosomes ${ }^{16}$. This model, however, is likely incomplete because it predicts that long-lived hotspots will be restricted to short chromosomes. Instead, long-lived hotspots are also observed on long chromosomes ${ }^{23}$.

Here, we show that most long-lived hotspots are located within large chromosome end-adjacent regions (EARs) that retain Hop1 and DSB markers in late prophase. Establishment of Hop1 enrichment in EARs requires Pch2, which preferentially removes Hop1 from interstitial chromosomal sequences, and is modulated by the histone deacetylase Sir2 and the nucleoporin Nup2. As EAR lengths are similar between chromosomes, EARs comprise a proportionally larger fraction of short chromosomes. We propose that the spatial bias in Hop1 enrichment increases relative DSB activity on shorter chromosomes and at least partially explains the increased recombination density on short chromosomes.

\section{Results}

Continued DSB formation is linked to chromosomal position. To identify features distinguishing short-lived and long-lived hotspots, we expanded the number of hotspots whose lifespan has been classified using Southern blot assays. To exclude dampening of DSB activity because of prophase exit ${ }^{14}$, we deleted the NDT80 gene, which encodes a transcription factor necessary for initiating the prophase exit program ${ }^{39}$. ndt80 $\Delta$ mutants halt meiotic progression at late prophase with fully synapsed chromosomes (pachynema) and extend the permissive time window for DSB formation 11,31 , allowing more efficient capture of long-lived hotspots. Southern analysis of $n d t 80 \Delta$ cells undergoing a synchronous meiotic time course revealed additional examples of long-lived $(Y O L 081 \mathrm{~W})$ and short-lived hotspots (YER004W, YER024W, YOL001W; Fig. 1a, Supplementary Fig. 1a), indicating that both hotspot classes are common in the yeast genome.

Plotting the positions of these and previously published hotspots analyzed in $n d t 80 \Delta$ mutants revealed that the differences in temporal regulation correlated closely with distance from telomeres. Whereas short-lived hotspots were located interstitially on chromosomes, long-lived hotspots were primarily found in large domains adjacent to chromosome ends (Fig. 1b). These data suggest that continued hotspot activity is linked to chromosomal position.

To extend this analysis across the genome, we assessed markers of DSB formation by ChIP-seq assay. Histone H2A phosphorylated on serine $129(\gamma-\mathrm{H} 2 \mathrm{~A})$, the homologue of mammalian $\gamma$ $\mathrm{H} 2 \mathrm{AX}$, is a well-documented chromatin modification that is activated by DSB formation and spreads into an approximately $50-\mathrm{kb}$ region around DNA breaks ${ }^{40,41}$. Samples were collected from synchronous $n d t 80 \Delta$ cultures at time points corresponding to early prophase $(T=3 \mathrm{~h})$ and late/extended prophase $(T=6 \mathrm{~h})$, followed by deep sequencing of $\gamma-\mathrm{H} 2 \mathrm{~A}$ chromatin immunoprecipitate. These analyses showed that $\gamma-\mathrm{H} 2 \mathrm{~A}$ is distributed relatively evenly along chromosomes in early prophase, with particular enrichment at meiotic axis sites but depletion at DSB hotspots (Supplementary Fig. 1b-d). In late prophase, however, $\gamma$-H2A enrichment was strongly biased towards the ends of all 16 chromosomes (Fig. 1c). This enrichment was most pronounced within $20-110 \mathrm{~kb}$ from telomeres (Fig. 1d). We refer to these regions as chromosome end-adjacent regions (EARs). Averaging across all EARs revealed that this spatial bias was also apparent in early prophase, albeit to a lesser extent (Fig. 1c (inset) and d). At both time points, $\gamma$-H2A enrichment in the EARs was above the 
a

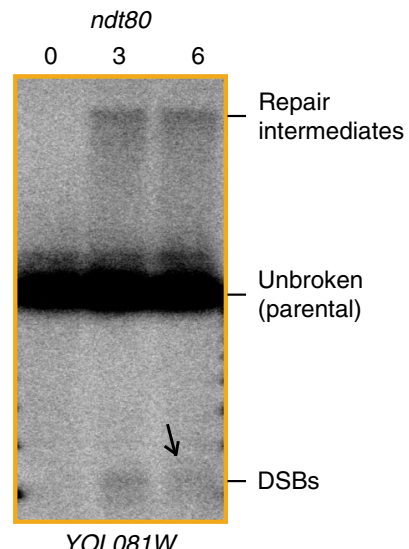

b

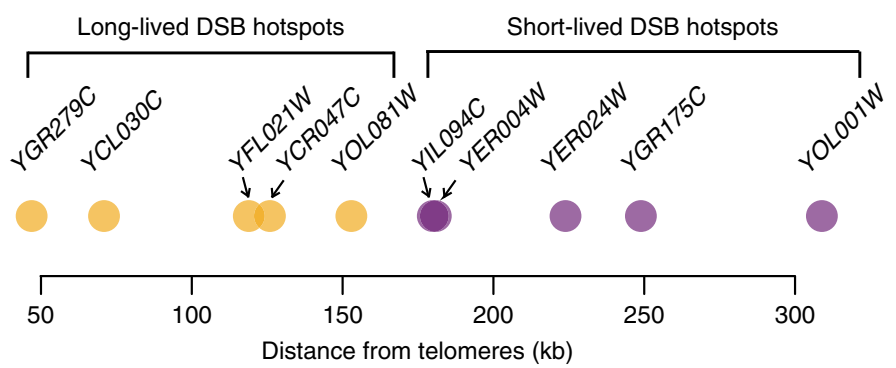

c
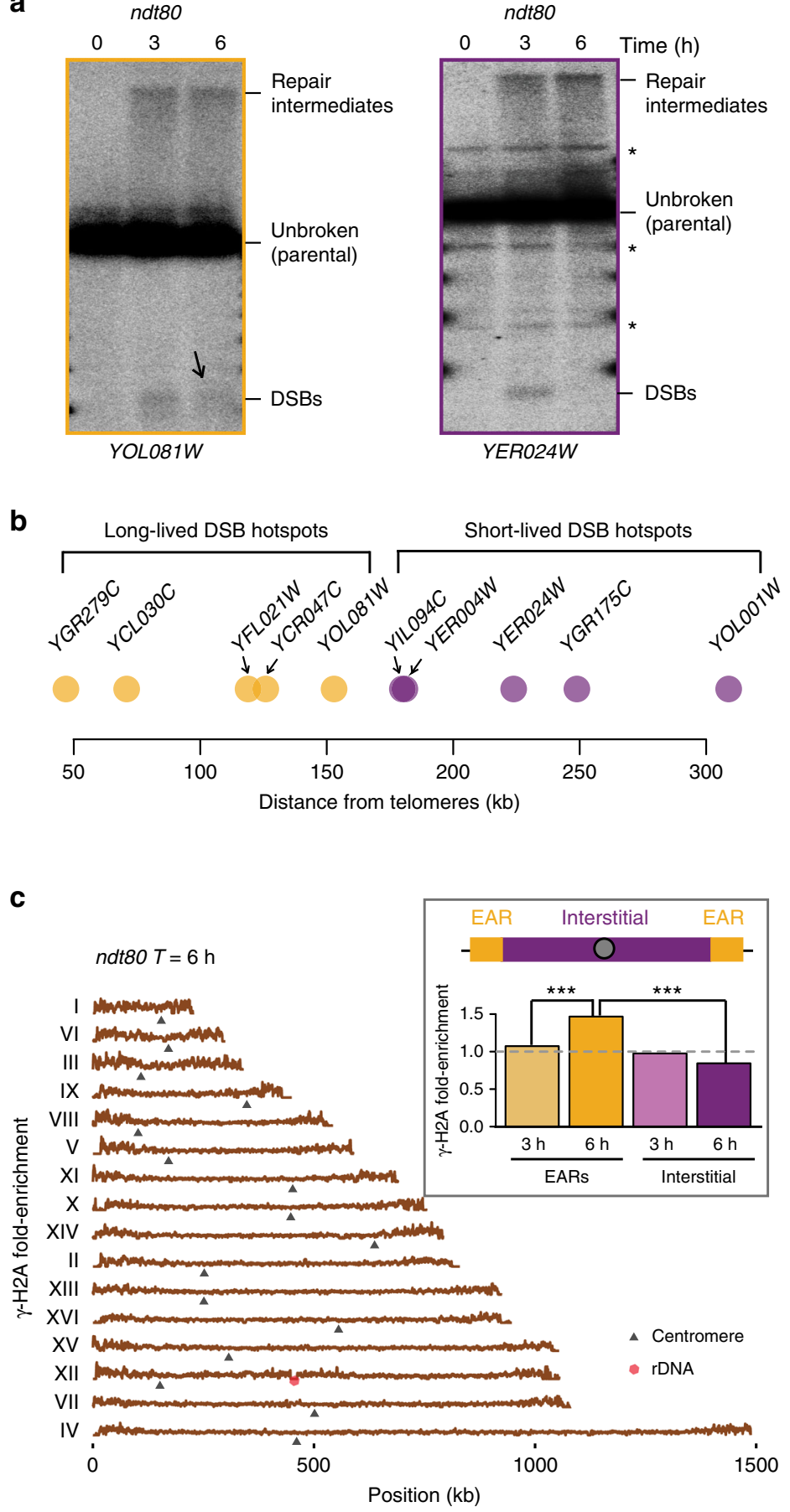

YER024W d

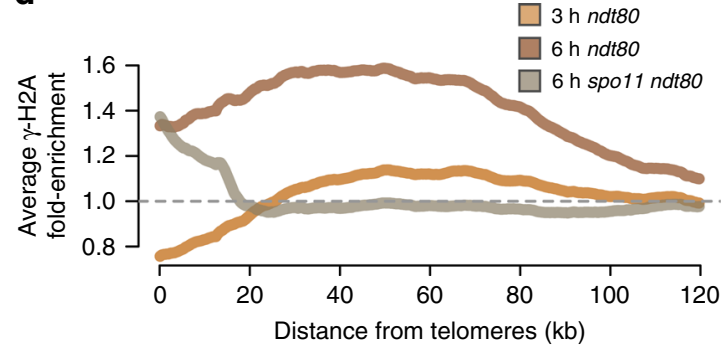

e
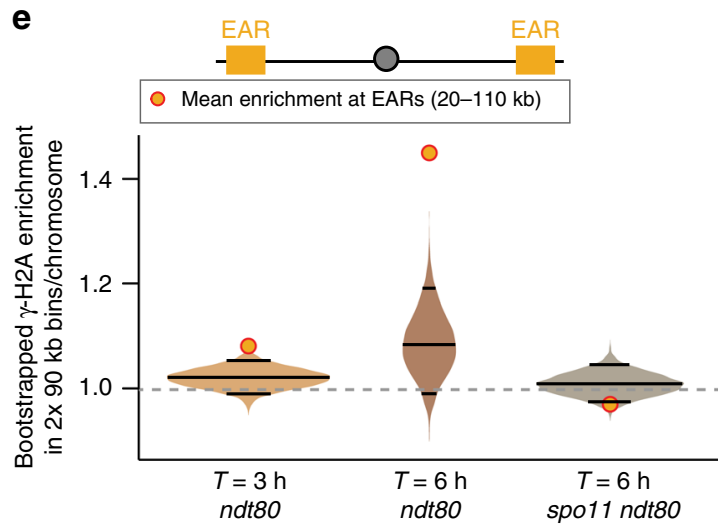

f
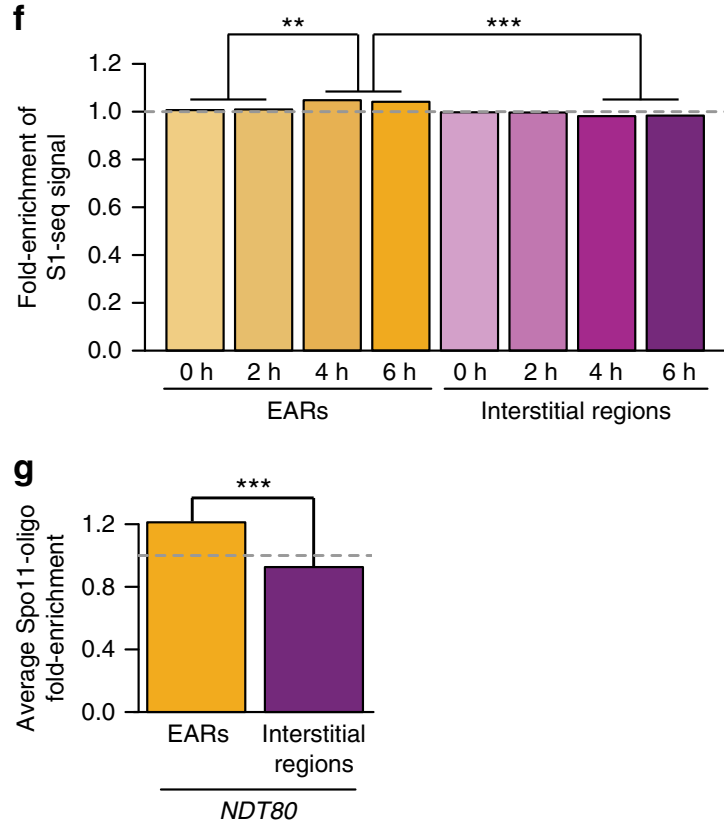

Fig. 1 Long-lived DSB hotspots occur primarily in EARs. a Southern analysis of DSBs from ndt80A cells progressing synchronously though meiotic prophase at YOLO81W (long-lived DSB hotspot, orange outline) and YERO24W (short-lived DSB hotspot, magenta outline). Black arrow highlights continued DSBs in late prophase $(T=6 \mathrm{~h})$ at the YOLO81W hotspot. Asterisk $\left({ }^{\star}\right)$ denotes nonspecific bands. b Distance of queried DSB hotspots (orange, long-lived; magenta, short-lived) from their closest telomere (11,23,31; this manuscript). c $\gamma$-H2A ChIP-seq enrichment is plotted along each of the 16 yeast chromosomes; black triangles mark the centromeres and the red hexagon marks the rDNA locus. The data are normalized to a global mean of 1 . Inset shows mean enrichment at EARs (20-110 kb from telomeres; orange) and interstitial chromosomal regions ( $>110 \mathrm{~kb}$ from telomeres; magenta) in early prophase $(T=3 \mathrm{~h})$ and extended prophase $(T=6 \mathrm{~h}) .{ }^{\star \star \star} P<0.001$, Mann-Whitney-Wilcoxon test. d Mean $\gamma$-H2A enrichment in the EARs (32 domains) is plotted as a function of distance from telomeres. e Bootstrap-derived distributions within two 90-kb bins per chromosome from ChIP-seq data are shown as violin plots. Lower and upper quantile $(95 \% \mathrm{Cl})$, as well as the median computed from the bootstrap data are depicted as horizontal lines. The orange/red dot shows the mean ChIP-seq enrichment in EARs (20-110 kb) for the respective samples. Divergence of the boot-strap derived median from the genome average is the result of data structure and large bin sizes. $\mathbf{f}$ Time series of S1-seq signal reflecting resected DSB ends ${ }^{43}$ are normalized to genome average and plotted as mean signal in EARs (20-110 kb, 32 domains) and interstitial chromosomal regions ( $>110 \mathrm{~kb}$ from either end of all chromosomes, 16 domains). ${ }^{\star \star \star} P<0.001$ and ${ }^{\star} * P<0.01$, ANOVA on mean enrichment followed by a post-hoc Tukey test. $\mathbf{g}$ Spo11-oligos within hotspots ${ }^{16,44}$ are normalized to genome average and plotted as mean signal in EARs (20-110 kb, 32 domains) and interstitial chromosomal regions (>110 kb from either end of all chromosomes, 16 domains). ${ }^{\star \star \star} P<0.001$, ANOVA on mean enrichment followed by a post-hoc Tukey test. The gray dashed line in $\mathbf{c}-\mathbf{g}$ is the genome average 
95\% confidence interval (CI) of a bootstrap-derived distribution (Fig. 1e).

$\gamma$-H2A enrichment near telomeres was largely dependent on SPO11, indicating that these regions experience enhanced meiotic DSB activity (Fig. 1d, e). Consistently, ChIP-seq analysis of Rad51, a DSB repair protein, also showed an enrichment of signal in EARs during the extended prophase in ndt80 $\Delta$ mutants (Supplementary Fig. 1e, f). We note that $\gamma$-H2A enrichment persisted within $20 \mathrm{~kb}$ from telomeres in spo11 10 mutants, in line with previous observations showing DSB-independent enrichment in these regions in mitotic cells ${ }^{42}$. These observations suggest that DSB activity in EARs is prolonged relative to genome average.

To assess DSB activity in EARs in wild-type cells (NDT80), we analyzed published genome-wide S1-seq datasets ${ }^{43}$. S1-seq measures unrepaired DNA ends and S1 nuclease-sensitive repair intermediates and thus also reports on DSB occurrence. S1-seq signal became significantly enriched in EARs over time compared to interstitial chromosomal sequences (Fig. 1f), closely mirroring the temporal enrichment of $\gamma-\mathrm{H} 2 \mathrm{~A}$ and $\operatorname{Rad} 51$ in these regions. This trend remained even after excluding the three shortest chromosomes, which consist primarily of EARs (Supplementary Fig. 1g). Analysis of available datasets ${ }^{16,44}$ further showed that Spo11-oligonucleotides (Spo11-oligos), a byproduct of DSB formation, are also derived from EARs at significantly higher levels compared to telomere-distal regions $(T=4 \mathrm{~h}$; Fig. $1 \mathrm{~g}$, Supplementary Fig. 1h). Together, these data indicate that hotspots located in EARs are partially refractory to DSB downregulation in late prophase.

Continued DSB formation correlates with Hop1 enrichment. We sought to identify regulators mediating differential DSB activity in late prophase. DSB correlates well with the presence of Hop1 on meiotic chromosomes observed by immunofluorescence and genome-wide assays $7,8,10,23$. Therefore, we monitored the evolution of Hop1 enrichment on wild-type (NDT80) meiotic chromosomes by ChIP-seq (Supplementary Fig. 2a-c). At the time of pre-meiotic DNA replication $(T=2 \mathrm{~h})$, Hop1 was enriched in large domains $(\sim 100 \mathrm{~kb})$ around the centromeres $(>95 \%$ CI; Fig. 2a, Supplementary Fig. 2a, d), likely predicting the early enrichment of Spo11 in these regions ${ }^{45}$. By early prophase $(T=3$ h), Hop1 enrichment became more distributed and formed peaks of enrichment along all chromosomes (Supplementary Fig. 2b), matching previously defined sites of enrichment ${ }^{10}$. Importantly, by mid/late prophase $(T=4 \mathrm{~h})$, Hop1 enrichment trended towards the EARs (Fig. 2b, Supplementary Fig. 2c). The increase of Hop1 enrichment in EARs was even more prominent in the extended prophase of $n d t 80 \Delta$-arrested cells ( $T=6 \mathrm{~h}$; Figs $2 \mathrm{c}, \mathrm{d}$ ). Consistently, in both wild type and ndt80 $\Delta$ cells, the enrichment of Hop1 in the EARs at the later time points was above 95\% CI for a bootstrap-derived distribution of enrichment along the genome (Fig. 2e). To determine whether Hop1 binding increased in the EARs or decreased in the interstitial regions, we performed spike-in normalization between ChIP-seq samples (SNP-ChIP ${ }^{46}$ ). This analysis showed a $32 \%$ reduction in Hop1 binding in the extended prophase of $n d t 80 \Delta$-arrested cells compared to the early prophase sample (Fig. 2f), indicating that Hopl enrichment in the EARs arises primarily from a depletion of Hop1 from interstitial regions during late prophase (Supplementary Fig. 2e). These data imply that continued DSB formation results from persistent Hop1 binding in the EARs.

To test if redistribution of Hop 1 in late prophase reflects an overall reorganization of the meiotic chromosome axis ${ }^{27}$, we analyzed enrichment of the chromosome axis factor Red1 by ChIP-seq (Supplementary Fig. 3a). Similar to Hop1, Red1 enrichment in EARs became more prominent in late prophase in $n d t 80 \Delta$ mutants (Supplementary Fig. 1b). Red1 enrichment in EARs was above the 95\% CI compared to a bootstrap-derived enrichment along the genome (Supplementary Fig. 3c) and significantly different from enrichment at telomere-distal regions (Supplementary Fig. 3a, inset). The persistent enrichment of axis proteins in the EARs suggests that meiotic chromatin remains poised for DSB formation in these regions during late prophase.

Because Hop1 recruits Mek1 kinase to meiotic chromosomes in response to DSB-induced checkpoint activation ${ }^{47,48}$, we also assessed Mek1 enrichment by ChIP-seq analysis in $n d t 80 \Delta$ cells (Supplementary Fig. 3d). Mek1 was enriched along chromosomes in early prophase with specific enrichment at sites of axis-protein binding, but also at DSB hotspots (Supplementary Fig. 4a-d), consistent with Mek1-dependent histone H3 T11 phosphorylation at DSB sites ${ }^{49}$. Additionally, Mek1 was enriched at centromeres and tRNA genes (Supplementary Fig. 4e, f). Mek1 enrichment at axis sites persisted into late prophase, whereas enrichment at hotspots was somewhat diminished, likely reflecting a global reduction in DNA breakage in late prophase (Supplementary Fig. 4b, d). Importantly, Mek1 enrichment was significantly enhanced in the EARs in late prophase (Supplementary Fig. $3 \mathrm{c}-\mathrm{e}$ ), providing further support that DSBs continue to form in these regions.

EAR-like regions flanking the ribosomal DNA. In addition to the EARs, Hop1 enrichment in late prophase also increased in $\sim 100-\mathrm{kb}$ regions flanking the repetitive ribosomal DNA (rDNA) locus on chromosome XII (Fig. 2c, red hexagon). This increased enrichment was above the $95 \%$ CI of a bootstrap-derived distribution in $n d t 80 \Delta$ samples (Fig. $2 \mathrm{~g}$ ). A similar trend, albeit below the $95 \% \mathrm{CI}$, was observed in wild-type (NDT80) cultures. Similar to EARs, the rDNA-adjacent Hop1 enrichment was accompanied by a significant local increase in $\gamma$-H2A signal (Supplementary Fig. 5a). Mek1 enrichment also followed this trend but was below the 95\% CI. To test if $\gamma$-H2A enrichment reflected continued breakage of DNA near the rDNA, we measured DSB activity at the rDNA-adjacent YLR152C locus using Southern analysis. Although rDNA-adjacent hotspots are generally weak ${ }^{50}$, analysis in an $n d t 80 \Delta$ background revealed that DSBs and repair intermediates increase throughout the time course at YLR152C (Supplementary Fig. 5b, c), similar to longlived hotspots in EARs (Fig. 1a). These findings demonstrate ongoing DSB activity in late prophase near the rDNA and suggest that the rDNA-adjacent regions, like EARs, escape negative feedback regulation of DSBs.

Zip1 is equally present in EARs and interstitial regions. Because cytological assays indicate that Hop1 is depleted from meiotic chromosomes upon SC assembly $23,27,28,51$, we asked if EARs are less likely to assemble an SC than interstitial chromosomal sequences. To this end, we surveyed localization of the SC protein Zip1 on late-prophase chromosomes in $n d t 80 \Delta$ samples by ChIP-seq (Fig. 3a). Consistent with previous reports, Zip1 was enriched around centromeres (Fig. 3b). However, enrichment of Zip1 in the EARs was not different from interstitial regions $(P=$ 0.564, Mann-Whitney-Wilcoxon test; Fig. 3a (inset), c, d). These findings suggest that Zip1 assembly on chromosomes is not sufficient for the spatial regulation of Hop1 in late prophase and that EARs remain enriched for Hop1 despite the presence of Zip1 in these regions.

Pch2 is required for late-prophase EAR enrichment of Hop1. The $\mathrm{AAA}^{+}$-ATPase Pch2 is recruited to synapsed chromosomes in an SC-dependent manner and is responsible for removal of 
a

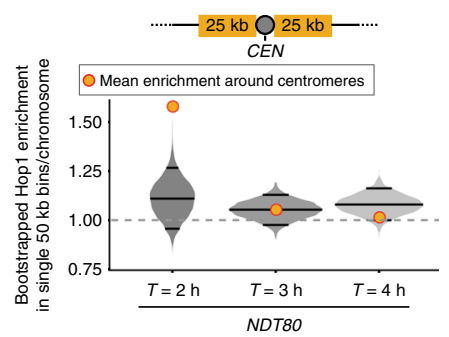

b

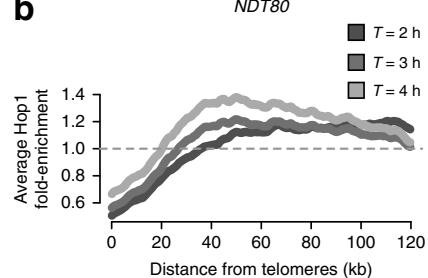

C

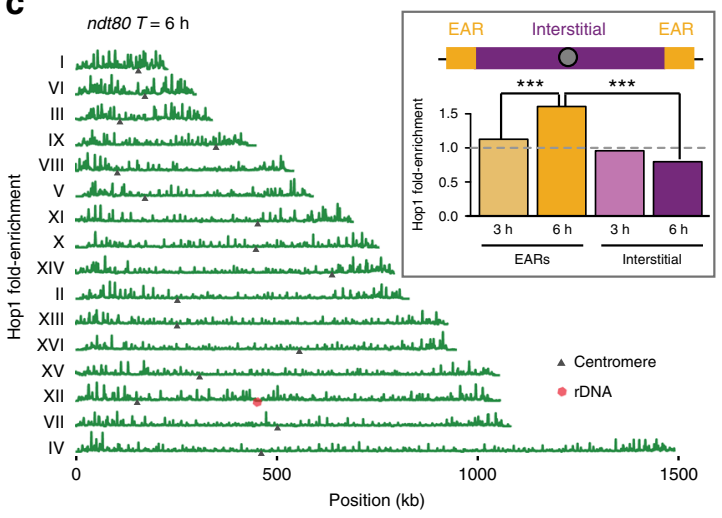

d
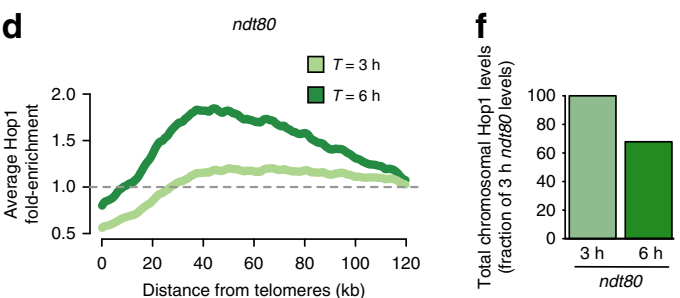

e

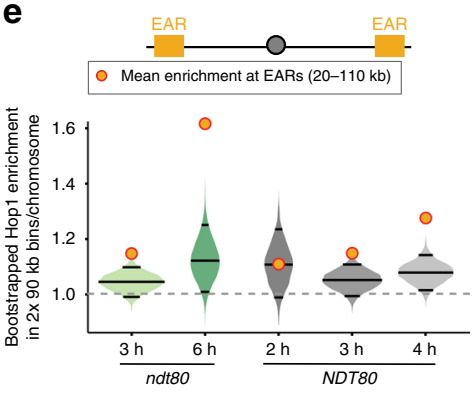

g

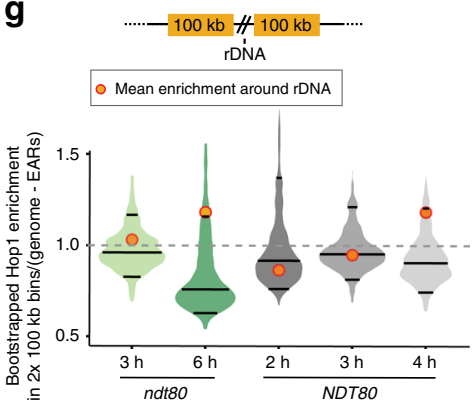

Fig. 2 Hop1 enrichment in EARs, centromere-proximal and rDNA-proximal regions during prophase. a Time series of Hop1 enrichment around centromeres $(50 \mathrm{~kb})$ in NDT80. Bootstrap-derived distributions within single 50-kb bins per chromosome are shown as violin plots and the horizontal lines within the plots represent the median and the two-ended 95\% Cls. The mean Hop1 ChIP-seq enrichment around centromeres (50 kb centered around centromeres) is shown as orange/red dots. b Mean Hop1 enrichment in the EARs (32 domains) is plotted as a function of distance from telomeres in NDT80. The gray dashed line is genome average. c Hop1 ChIP-seq enrichment (dark green) in ndt80 $\Delta$-arrested late prophase cells plotted along each of the 16 yeast chromosomes; black triangles mark the centromeres and the red hexagon marks the rDNA locus. The data are normalized to a global mean of 1 . Inset shows mean enrichment in EARs (20-110 kb, orange) and interstitial chromosomal regions (magenta) in early prophase $(T=3 \mathrm{~h})$ and extended prophase $\left(T=6 \mathrm{~h}\right.$ ). ${ }^{\star \star \star} P<0.001$, Mann-Whitney-Wilcoxon test. d Mean Hop1 enrichment in the EARs (32 domains) is plotted as a function of distance from telomeres in $n d t 80 \Delta$ cells. e Bootstrap-derived distributions within two 90-kb bins per chromosome from Hop1 ChIP-seq data depicted as violin plots. The horizontal lines in the violin plots represent the median and the two-ended 95\% Cls. The mean Hop1 ChIP-seq enrichment in EARs (20-110 kb) for the respective samples is shown as orange/red dots. $\mathbf{f}$ Spike-in normalized total chromosomal Hop1 levels in ndt80 samples during extended prophase ( $T=6 \mathrm{~h}$ ) compared to early prophase $(T=3 \mathrm{~h}) . \mathbf{g}$ Bootstrap-derived distributions within two 100-kb bins per genome from Hop1 ChIP-seq data illustrated as violin plots. The horizontal lines in the violin plots represent the median and the two-ended Cls. The mean Hop1 ChIP-seq enrichment in rDNA-adjacent domains (100 kb on either side of the rDNA) for the respective samples is shown as orange/red dots

Hop1 from chromosomes ${ }^{23,27,28}$. To test if Pch2 is responsible for establishing the Hop1-enriched EARs in late prophase, we determined Hop1 binding in synchronous pch $2 \Delta$ ndt80 $\Delta$ cultures by ChIP-seq. These analyses showed pervasive binding of Hop1 along chromosomes into late prophase, consistent with the persistent cytological signal and the elevated Hop1 protein levels in pch $2 \Delta$ mutants (Fig. 4a) 23,27,28,52. Hop1 dropped significantly below genome average in the EARs in the absence of $\mathrm{PCH} 2$ (Figs 4b, c). Spike-in normalization between ChIP-seq samples revealed a slight reduction in Hopl enrichment in early prophase in the pch $2 \Delta n d t 80 \Delta$ mutants but a $25.7 \%$ increase during the extended prophase compared to early prophase $n d t 80 \Delta$ samples (Fig. 4d). These data show that Hop1 specifically accumulates in the interstitial regions in the absence of $\mathrm{PCH} 2$, whereas binding levels in EARs remains largely unchanged. The altered Hop1 enrichment was reflected in altered DSB distribution and dynamics. Hotspots in interstitial regions continued to break and accumulate repair intermediates in pch $2 \Delta$ ndt80 $\Delta$ cultures, whereas hotspots in EARs exhibited comparatively reduced activity (Fig. 4e, Supplementary Fig. 6a, b). Consistently, Spo11oligo counts from $p c h 2 \Delta$ mutants were not significantly different between EARs and interstitial regions ( $T=4 \mathrm{~h}$; Fig. $4 \mathrm{f})$. These findings suggest that Pch2 promotes Hop1 removal from interstitial regions, leading to relative Hopl enrichment and DSB activity in the EARs in late prophase.
Pch2 suppresses DSBs at rDNA borders and centromeres. In addition to the broad effect on interstitial regions, several genomic landmarks were particularly affected by the loss of $\mathrm{PCH} 2$. As previously reported ${ }^{50}$, Hop1 enrichment in pch $2 \Delta$ mutants was increased around the rDNA array, resulting in elevated DSB levels in these regions (Supplementary Fig. 6c, d). In the extended prophase of $n d t 80 \Delta$ mutants, Hop1 ChIP enrichment was further enhanced and DSBs continued to form near the rDNA (Supplementary Fig. 6c-e).

Hop1 enrichment was also strongly elevated in the immediate vicinity of centromeres in the absence of $\mathrm{PCH} 2$ (Fig. 5a). This increase was already detectable above the 95\% CI in early prophase and became more pronounced in late prophase (Fig. 5b). Accordingly, Southern analysis of a centromeric hotspot $(Y O L 001 \mathrm{~W})$ revealed elevated DSB activity in pch $2 \Delta n d t 80 \Delta$ mutants in late prophase (Figs $5 \mathrm{c}, \mathrm{d}$ ). The increase in DSBs was not due to stalled repair because it was accompanied by an increase in repair intermediates (Fig. 5c). Likewise, Spo11-oligos were significantly elevated around centromeres (6-kb region; $P=$ 0.02 , Mann-Whitney-Wilcoxon test) and in the $40-\mathrm{kb}$ pericentromeric regions $(P=0.026$, Mann-Whitney-Wilcoxon test) in pch $2 \Delta$ mutants compared to wild type (Supplementary Fig. 6f). These data indicate that Pch2 is required to restrict Hop1-linked DSB activity not only around the rDNA but also around centromeres. 
a

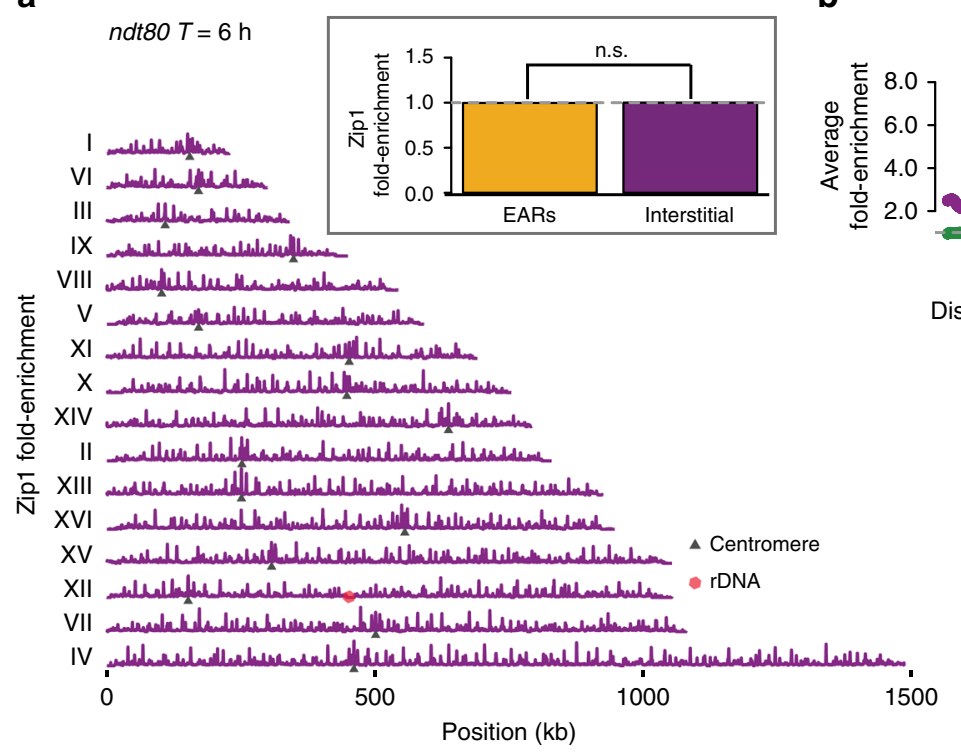

C

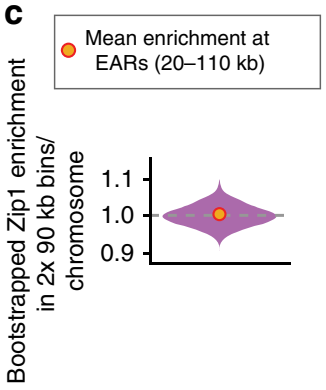

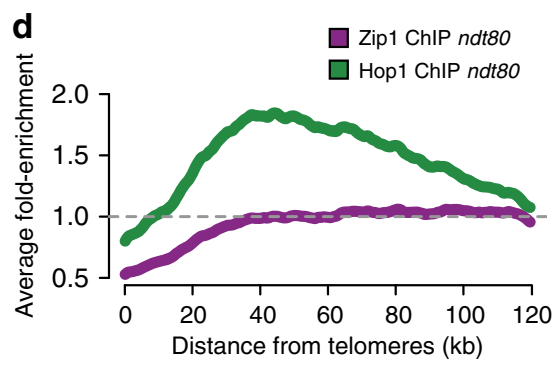

Fig. $3 \mathrm{Zip} 1$ is not depleted from EARs. a Zip1 ChIP-seq enrichment (magenta) in ndt804-arrested late prophase cells plotted along each of the 16 yeast chromosomes; black triangles mark the centromeres and the red hexagon marks the rDNA locus. The data are normalized to a global mean of 1 . Inset shows mean enrichment in EARs (20-110 kb, orange) and interstitial chromosomal regions (magenta) in extended prophase $(T=6 \mathrm{~h}) . P=0.564$ (n.s.), Mann-Whitney-Wilcoxon test. b Zip1 (magenta) and Hop1 (dark green) ChIP-seq enrichment around centromeres from ndt80 $\Delta$-arrested cultures in late prophase $(T=6 \mathrm{~h})$. c Bootstrap-derived distributions within two $90-\mathrm{kb}$ bins per chromosome from Zip1 ChIP-seq data are depicted as a violin plot. The horizontal lines in the violin plot represent the median and the two-ended 95\% Cls. The mean Zip1 ChIP-seq enrichment in EARs (20-110 kb) is shown as orange/red dots. $\mathbf{d}$ Hop1 (dark green) and Zip1 (magenta) ChIP-seq enrichment in late prophase $(T=6 \mathrm{~h}$ ) is plotted as a mean enrichment in the EARs (32 domains) as a function of the distance from telomeres. The gray dashed line is genome average

The nucleoporin Nup2 promotes Pch2 binding to chromosomes. We sought to identify additional regulators that drive Hop1 enrichment in the EARs. The disruption of several telomeric factors, including the tethering factor ESC1, the telomerelength regulator TEL1, or the silencing factor SIR3 did not significantly affect enrichment of Hop1 in the EARs (Supplementary Fig. 7a). Deletion of the meiotic telomere-clustering factor NDJ1 severely disrupted Hop1 enrichment in EARs, while conditional depletion caused only slight effects (Supplementary Fig. 7a, b). However, the interpretation of these results is complicated by the fact that loss of NDJ1 also causes synapsis defects ${ }^{53}$. As the nonessential nucleoporin Nup2 was recently identified as an interactor of $\mathrm{Ndj} 1^{54}$, we also analyzed Hop 1 in nup $2 \Delta$ mutants. Intriguingly, Hop1 did not become enriched in the EARs in late prophase in nup2 $2 \Delta d t 80 \Delta$ mutants (Fig. 6a-c). This effect, however, was due to diminished Hop1 removal from interstitial regions, which continued to exhibit prominent Hop1 peaks in late prophase (Fig. 6a), similar to pch $2 \Delta$ mutants. Indeed, although the phenotypes are generally less pronounced than in a pch $2 \Delta$ mutant, loss of NUP2 also caused retention of Hop1 on synapsed chromosomal regions (Fig. 7a, Supplementary Fig. 7c), a pch2-like enrichment of Hop1 in the vicinity of centromeres (Fig. 6d), and increased DSB levels at interstitial hotspots (Supplementary Fig. 6a). These data suggest that NUP2 and $\mathrm{PCH} 2$ act in a common pathway.

A common pathway is supported by the fact that the Hop1 localization defects of the single mutants are non-additive. The nup $2 \Delta$ pch $2 \Delta$ double mutant resembled the pch $2 \Delta$ single mutant when Hop 1 accumulation was analyzed on chromosome spreads (Fig. 7a, Supplementary Fig. 7c). The fact that the double mutant phenocopies the pch $2 \Delta$ phenotype implies that $\mathrm{PCH} 2$ is epistatic to NUP2. Consistent with this interpretation, Pch2 foci on meiotic chromosomes are noticeably diminished in the absence of
NUP2, with most of the Pch2 concentrated in a bright nucleolar signal (Fig. 7b). Quantification of the nucleolar signal indicated that Pch2 is even more abundant in the nucleolus in nup2 $\Delta$ mutants than in wild type (Fig. 7c).

To investigate whether the nucleolar pool of Pch2 is functional in the absence of NUP2, we analyzed DSB formation and Hop1 enrichment near the rDNA. nup $2 \Delta$ mutants do not phenocopy pch $2 \Delta$ mutants for rDNA-associated phenotypes, as there is no DSB induction near the rDNA (Supplementary Fig. 6c, d). In fact, nup $2 \Delta$ mutants showed a relative decrease in Hop1 ChIP-seq signal near the rDNA compared to wild type (Supplementary Fig. 6e). These results imply that the nucleolar pool of Pch2 is fully functional in the absence of NUP2 and suggest that Nup2 is not a general activator of Pch2 function but rather acts through controlling the nuclear distribution of Pch2.

Nup2 regulation of Pch2 is mediated by Sir2. Nup2 may either promote the binding of Pch2 to synapsed chromosomes or suppress sequestration of Pch2 in the nucleolus. To distinguish between these possibilities, we analyzed mutants lacking the rDNA-enriched silencing factor SIR2, which is required for the nucleolar localization of $\mathrm{Pch} 2^{28}$. nup $2 \Delta$ sir $2 \Delta$ double mutants showed a complete loss of Pch2 from the nucleolus (Fig. 7b, lower panels), indicating that the nucleolar over-enrichment of Pch2 in nup2 $2 \Delta$ mutants is fully dependent on SIR2. Importantly, Pch2 signal on synapsed chromosomes was indistinguishable between sir $2 \Delta$ and sir $2 \Delta$ nup $2 \Delta$ double mutants (Fig. 7b, Supplementary Fig. 7d), showing that NUP2 does not promote binding of Pch2 to synapsed chromosomes. These data suggest that Nup2 counteracts Sir2-dependent recruitment of Pch2 to the nucleolus.

Consistent with this model, the aberrant Hop1 accumulation on synapsed chromosomes observed in nup $2 \Delta$ mutants is rescued 
a

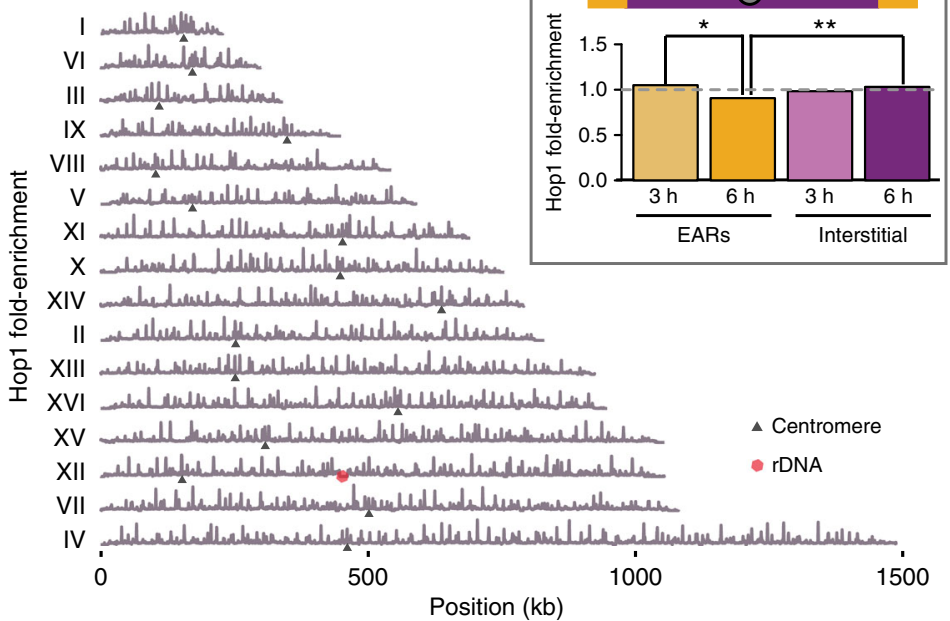

b

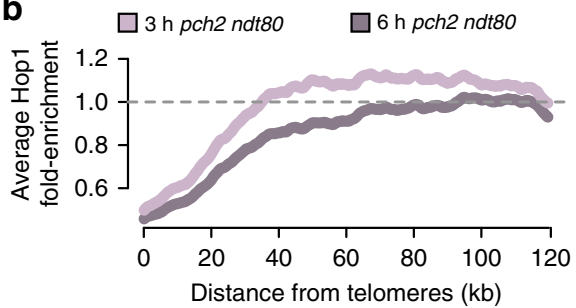

C

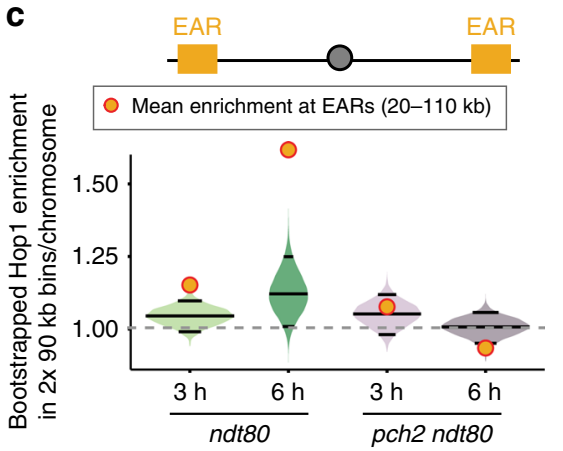

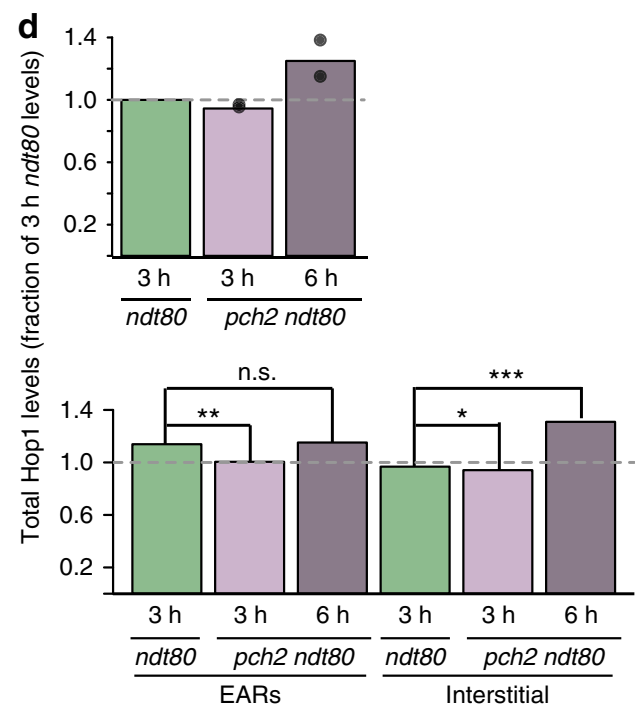

e
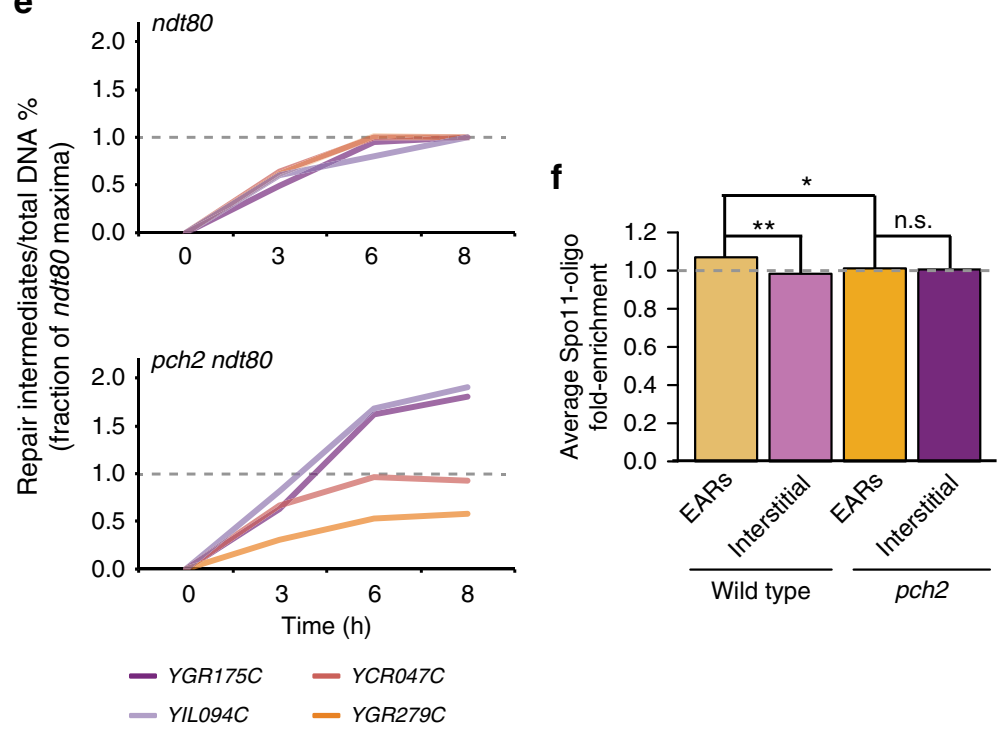

Fig. 4 Pch2 controls regional distribution of Hop1 and DSBs in EARs. a Hop1 ChIP-seq enrichment in pch $2 \Delta$ ndt80 late prophase ( $T=6 \mathrm{~h})$ cells plotted along each of the 16 yeast chromosomes; black triangles mark the centromeres and the red hexagon marks the rDNA locus. The data are normalized to a global mean of 1. Inset shows mean Hop1 enrichment in EARs (20-110 kb, orange) and interstitial chromosomal regions (magenta) in early prophase ( $T=$ $3 \mathrm{~h}$ ) and late prophase $(T=6 \mathrm{~h}) .{ }^{\star *} P<0.01$ and ${ }^{\star} P<0.05$, Mann-Whitney-Wilcoxon test. b Mean Hop1 enrichment in the EARs (32 domains) is plotted as a function of the distance from telomeres in pch2 $2 \Delta$ dt $80 \Delta$ at early $(T=3 \mathrm{~h})$ and late prophase $(T=6 \mathrm{~h})$. The gray dashed line is genome average. c Bootstrap-derived distributions within two $90-\mathrm{kb}$ bins per chromosome from Hop1 ChIP-seq data illustrated as violin plots for ndt80 $\Delta$ and pch2 $\mathrm{ndt} 80 \Delta$ cells. The horizontal lines in the violin plots represent the median and the two-ended 95\% Cls. The mean Hop1 ChIP-seq enrichment in EARs (20-110 kb) for the respective samples is shown as orange/red dots. The gray dashed line is genome average. $\mathbf{d}$ Spike-in normalized Hop1 enrichment in pch2 $2 \Delta$ nt $80 \Delta$ at $T=3 \mathrm{~h}$ and $T=6 \mathrm{~h}$ compared to early prophase $(T=3 \mathrm{~h}) \mathrm{ndt} 80 \Delta$ sample. Total Hop1 levels are depicted in the upper panel. The range in levels is depicted with black dots. The lower panel shows spike-in normalized Hop1 levels from early prophase $(T=3 \mathrm{~h})$ and late prophase $(T=6 \mathrm{~h}) p c h 2 \Delta$ ndt80 $\Delta$ samples plotted as mean enrichment in EARs (20-110 kb) and interstitial chromosomal regions compared to early prophase ndt80 sample. The gray dashed line is genome average of early prophase $n d t 80 \Delta$ sample. ${ }^{\star \star \star} P<0.0001$, ${ }^{\star \star} P<0.01,{ }^{\star} P<0.05$, and n.s. $P=0.32$, Mann-Whitney-Wilcoxon test. e Cumulative DNA breaks measured as percentage of repair intermediates over total DNA and depicted as a fraction of $n d t 80 \Delta$ at $T=8 \mathrm{~h}$ are shown for ndt804, and pch24 ndt804. Interstitial hotspots (YGR175C, YIL094C) are shown in shades of magenta and hotspots in EARs (YCR047C, YGR279C) are depicted in shades of orange. The data are averages of two independent biological replicates. $\mathbf{f}$ Spo11-oligo signals from wild-type control ${ }^{44}$ and pch2 $\Delta$ mutant are normalized to genome average and plotted as mean signal in EARs (20-110 kb, 32 domains) and interstitial chromosomal regions ( $>110 \mathrm{~kb}$ from either end of all chromosomes, 16 domains). The gray dashed line is the genome average. ${ }^{\star \star} P<0.01,{ }^{\star} P<0.1$, n.s. not significant, ANOVA on mean enrichment followed by a post-hoc Tukey test 


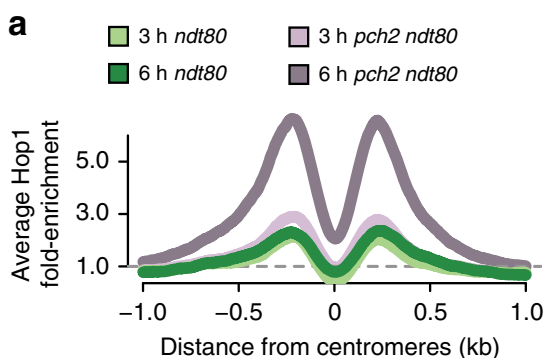

b

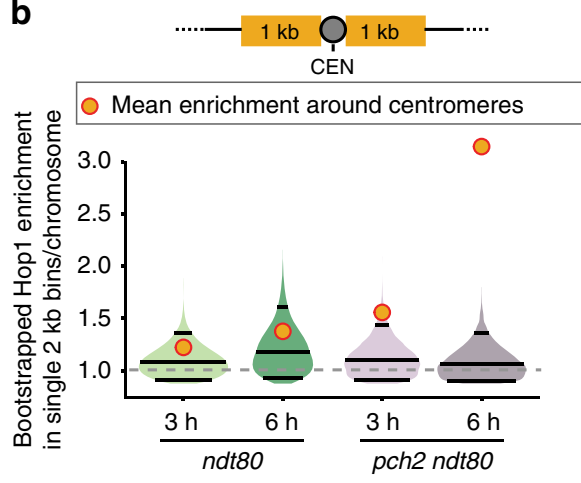

C
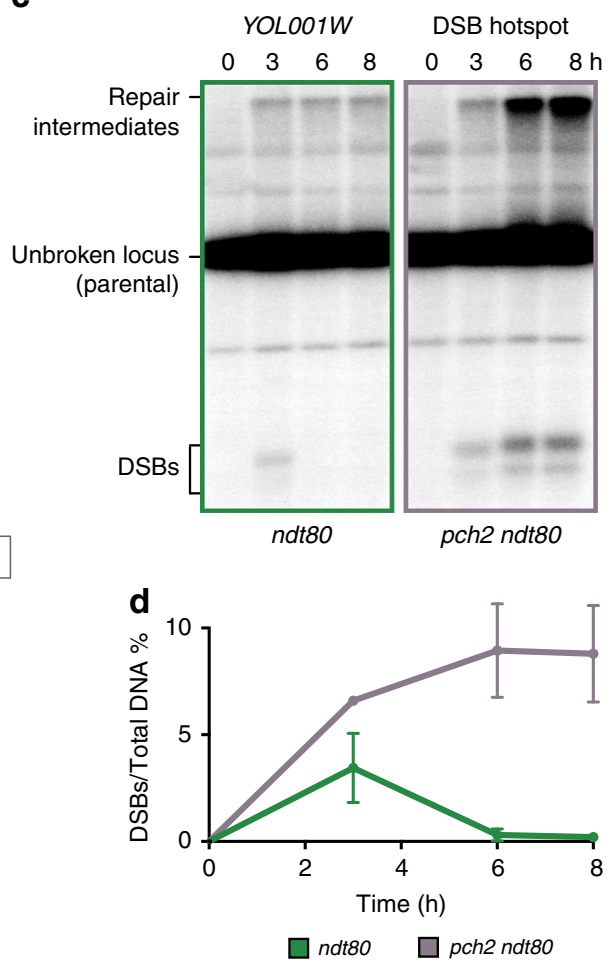

Fig. 5 Pch2 controls regional distribution of Hop1 and DSBs around centromeres. a Mean Hop1 enrichment around the centromeres normalized to genome

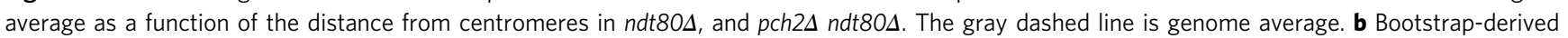
distributions within single $2-\mathrm{kb}$ bins per chromosome are illustrated as violin plots for Hop1 ChIP-seq data from ndt $80 \Delta$, and pch $2 \Delta$ ndt $80 \Delta$ mutants. The horizontal lines in the violin plots represent the median and the two-ended 95\% Cls. The mean Hop1 ChIP-seq enrichment around centromeres ( $2 \mathrm{~kb}$ ) for

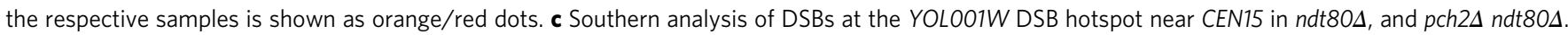
Percentage of DSBs over total DNA at the YOLO01W locus at the indicated time points is shown in $\mathbf{d}$. The data are mean of two independent biological replicates and error bars represent the range

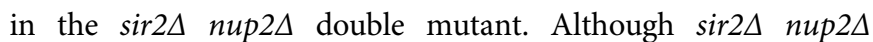
mutants have substantial synapsis defects, Hop1 was never observed on synapsed chromosome fragments (Fig. 7a), suggesting efficient Hop1 removal by Pch2. These data are consistent with the abundant presence of Pch2 on chromosomes in the double mutant and indicate that NUP2 acts upstream of SIR2 to control Hop1 localization.

To further investigate the role of Sir2 in controlling Hop1 distribution, we performed ChIP-seq of Hop1. This analysis showed that Hop1 was precociously enriched in the EARs in sir $2 \Delta$ ndt80 $\Delta$ mutants, with strong enrichment already detectable in early prophase $(T=3 \mathrm{~h}$ ) (Figs $6 \mathrm{c}, \mathrm{e}$ ). This pattern may reflect faster meiotic progression when Pch2 is overactive ${ }^{27}$. Consistent with an accelerated prophase, we noted a slightly faster appearance of fully synapsed chromosomes in sir $2 \Delta$ mutants (Supplementary Fig. 7e), as well as faster accumulation of repair intermediates, in particular for interstitial regions (Fig. 7d). This acceleration likely reflects the premature elimination of Mek1, which normally suppresses repair ${ }^{23,55}$. It is possible that faster progression also leads to a premature shutdown of DSB formation because Spo11-oligo analysis at $T=4 \mathrm{~h}$ shows comparable levels of DSB formation between EARs and interstitial regions in sir $2 \Delta$ mutant (Supplementary Fig. $7 \mathrm{f}$ ). In line with this possibility, repair intermediates do not continue to accumulate at later time points in sir $2 \Delta n d t 80 \Delta$ mutants (Fig. $7 \mathrm{~d}$ ). Taken together, our data implicate a regulatory pathway consisting of Nup2, Sir2, and Pch2 in driving the enrichment of
Hop1 in EARs and controlling the window of opportunity for DSB formation in interstitial regions (Fig. 7e).

Enrichment of Hop1 and DSB markers favors short chromosomes. The fact that EARs occupy a proportionally larger fraction of short chromosomes (Fig. 8a) provides a possible mechanism for increasing relative DSB levels on short chromosomes. Indeed, plotting $\gamma-\mathrm{H} 2 \mathrm{~A}$ enrichment per $\mathrm{kb}$ as a function of chromosome size revealed a distinct, SPO11-dependent over-enrichment of $\gamma$ $\mathrm{H} 2 \mathrm{~A}$ on short chromosomes in late prophase (Fig. 8b). Similarly biased enrichment was also observed for Hop 1 and Mek1 in early prophase and increased further in late prophase (Fig. 8c, Supplementary Fig. 8a). Moreover, Hopl enrichment increased on short chromosomes during prophase regardless of whether NDT80 was present (Supplementary Fig. 8b).

The early enrichment of Hop1 and Mek1 on short chromosomes may be driven by Red1, which recruits Hop1 to chromosomes ${ }^{51,56}$ and also exhibits chromosome size bias for enrichment in early prophase (Supplementary Fig. 8c) ${ }^{10}$. Supporting this model, the pattern of chromosome size bias between Red1 and Hop1 in early prophase was not significantly different (ANOVA, $P=0.167$ ). However, the Red1 chromosome size bias did not increase significantly between early and late prophase (Supplementary Fig. 8c), likely because Red1 experiences a less severe SC-dependent depletion than Hop1 in late prophase $^{51}$. Importantly, calculating the mean Hop1 enrichment per chromosome while excluding EARs significantly reduced the 
a

nup2 $n d t 80 T=6 \mathrm{~h}$

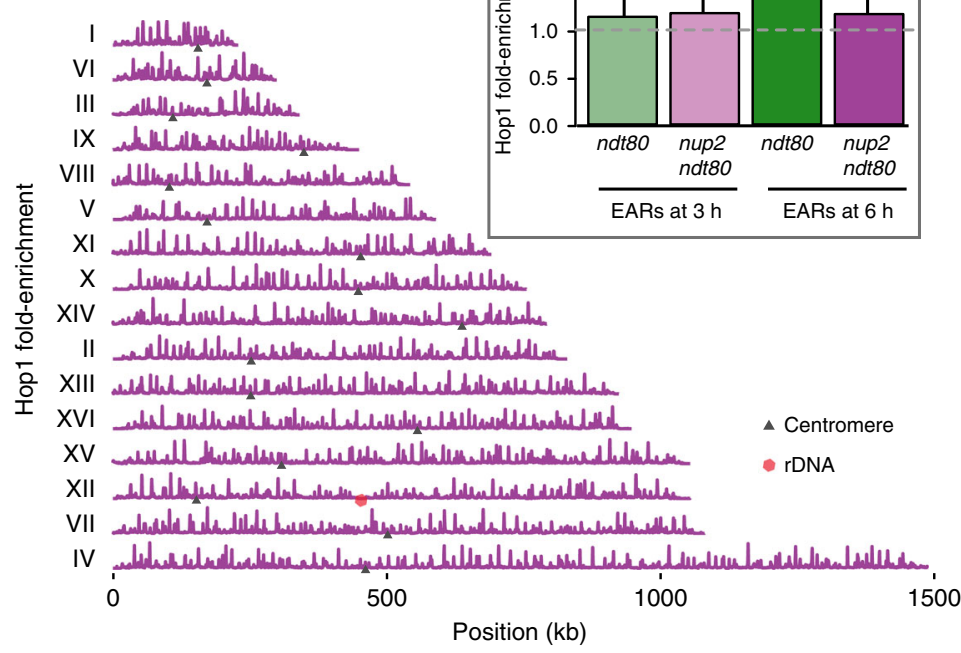

Position (kb)

e

$\operatorname{sir} 2 \mathrm{ndt80} T=3 \mathrm{~h}$

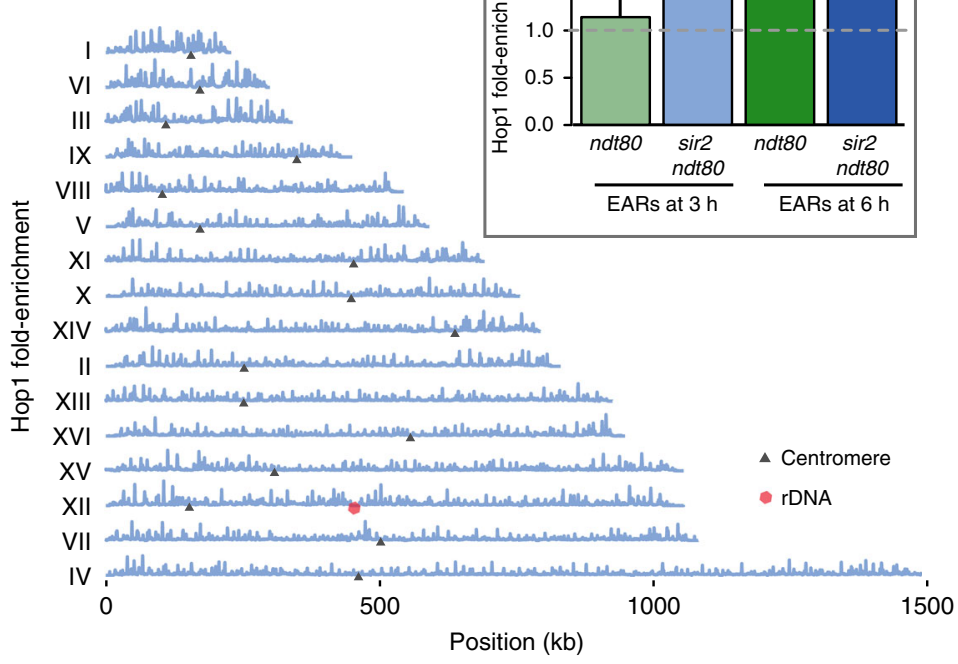

b

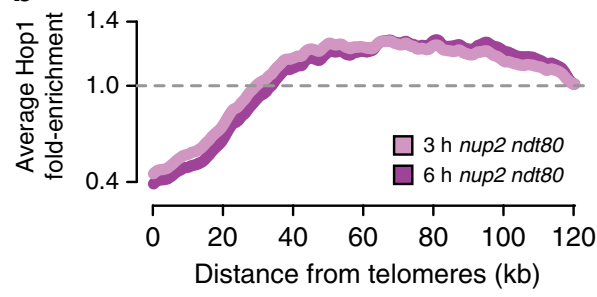

C
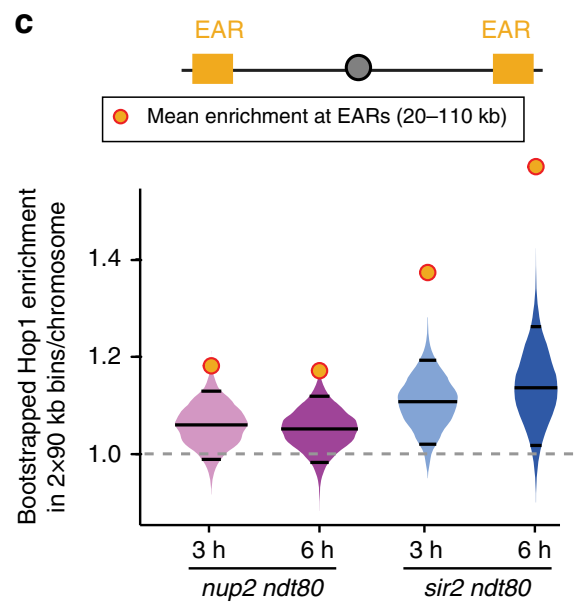

d
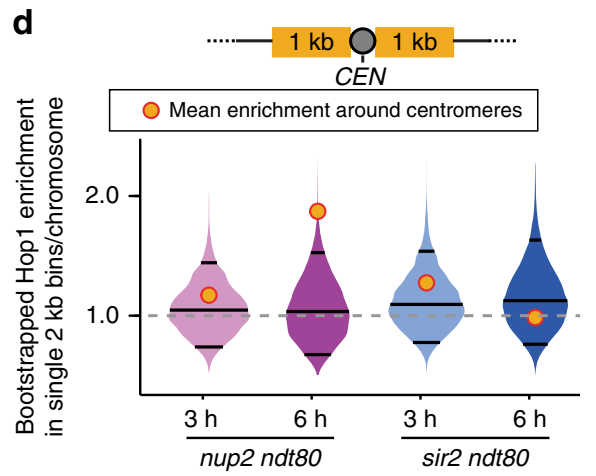

$\mathbf{f}$

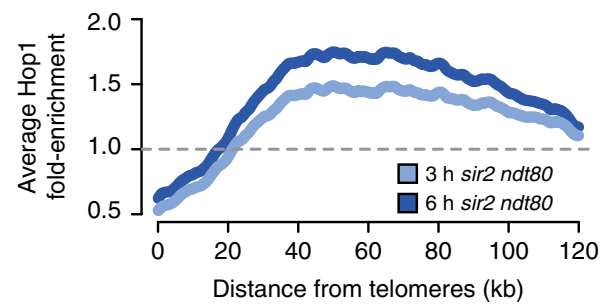

Fig. 6 Nup2 and Sir2 also regulate regional Hop1 distribution in prophase. a Hop1 ChIP-seq enrichment in late prophase cells ( $T=6 \mathrm{~h}$ ) from nup2 2 ndt80 $\Delta$ in magenta is plotted along each of the 16 yeast chromosomes; black triangles mark the centromeres and the red hexagon marks the rDNA locus. The data are normalized to a global mean of 1. Inset shows mean Hop1 enrichment in EARs (20-110 kb) in nup2 $\mathrm{ndt} 80 \Delta$ during early prophase ( $T=3 \mathrm{~h}$, pink) and late prophase ( $T=6 \mathrm{~h}$, magenta) compared to ndt80 $\Delta$ samples. ${ }^{\star \star} P<0.001$, and n.s. not significant, Mann-Whitney-Wilcoxon test. $\mathbf{b}$ Mean Hop1 enrichment in the EARs (32 domains) is plotted as a function of the distance from telomeres in nup2 $\Delta$ ndt80 4 . c Bootstrap-derived distributions within two

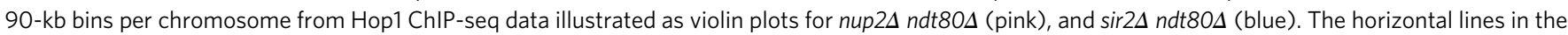
violin plots represent the median and the two-ended 95\% Cls. The mean Hop1 ChIP-seq enrichment in EARs (20-110 kb) for the respective samples is shown as orange/red dots. The gray dashed line is genome average. d Bootstrap-derived distributions within single 2-kb bins per chromosome are

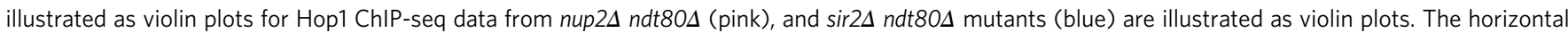
lines in the violin plots represent the median and the two-ended $95 \% \mathrm{Cls}$. The mean Hop1 ChIP-seq enrichment around centromeres ( $2 \mathrm{~kb}$ ) for the respective samples is shown as orange/red dots. e Hop1 ChIP-seq enrichment in early prophase cells ( $T=3 \mathrm{~h}$ ) from sir $2 \Delta$ ndt $80 \Delta$ in light blue is plotted along each of the 16 yeast chromosomes; black triangles mark the centromeres and the red hexagon marks the rDNA locus. The data are normalized to a global mean of 1. Inset shows mean Hop1 enrichment in EARs (20-110 kb) in sir $2 \Delta$ ndt80 $\Delta$ during early prophase ( $T=3 \mathrm{~h}$, light blue) and late prophase ( $T=6 \mathrm{~h}$, dark blue) compared to ndt80 $\Delta$ samples. ${ }^{\star \star} P<0.01$, and n.s. not significant, Mann-Whitney-Wilcoxon test. $\mathbf{f}$ Mean Hop1 enrichment in the EARs (32 domains) is plotted as a function of the distance from telomeres in sir2 $\mathrm{ndt} 80 \Delta$ 
a
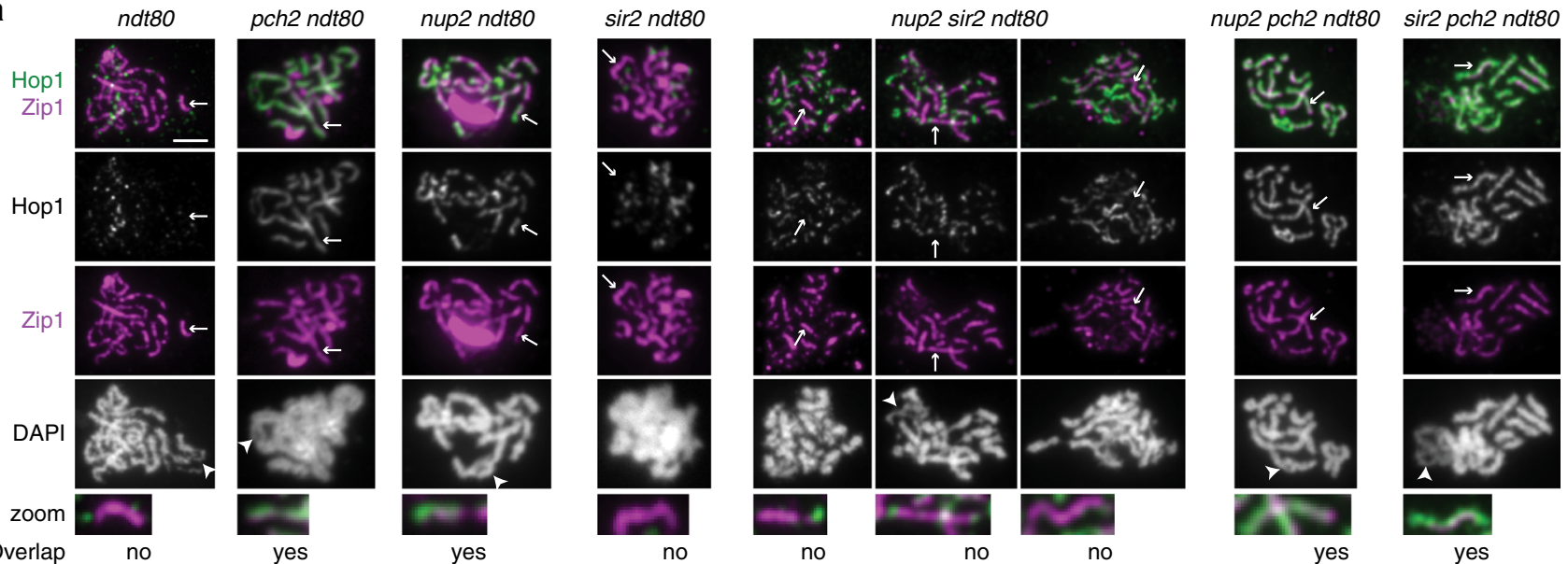

b
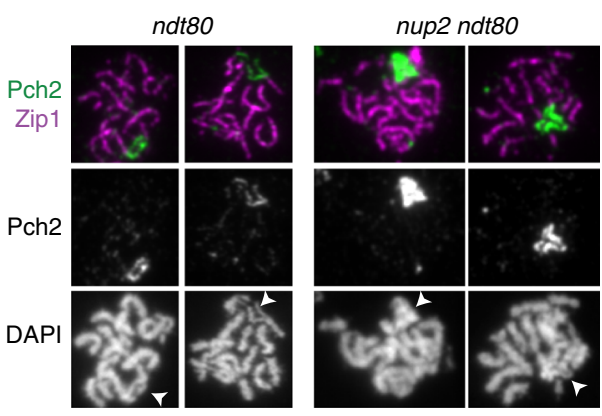

C
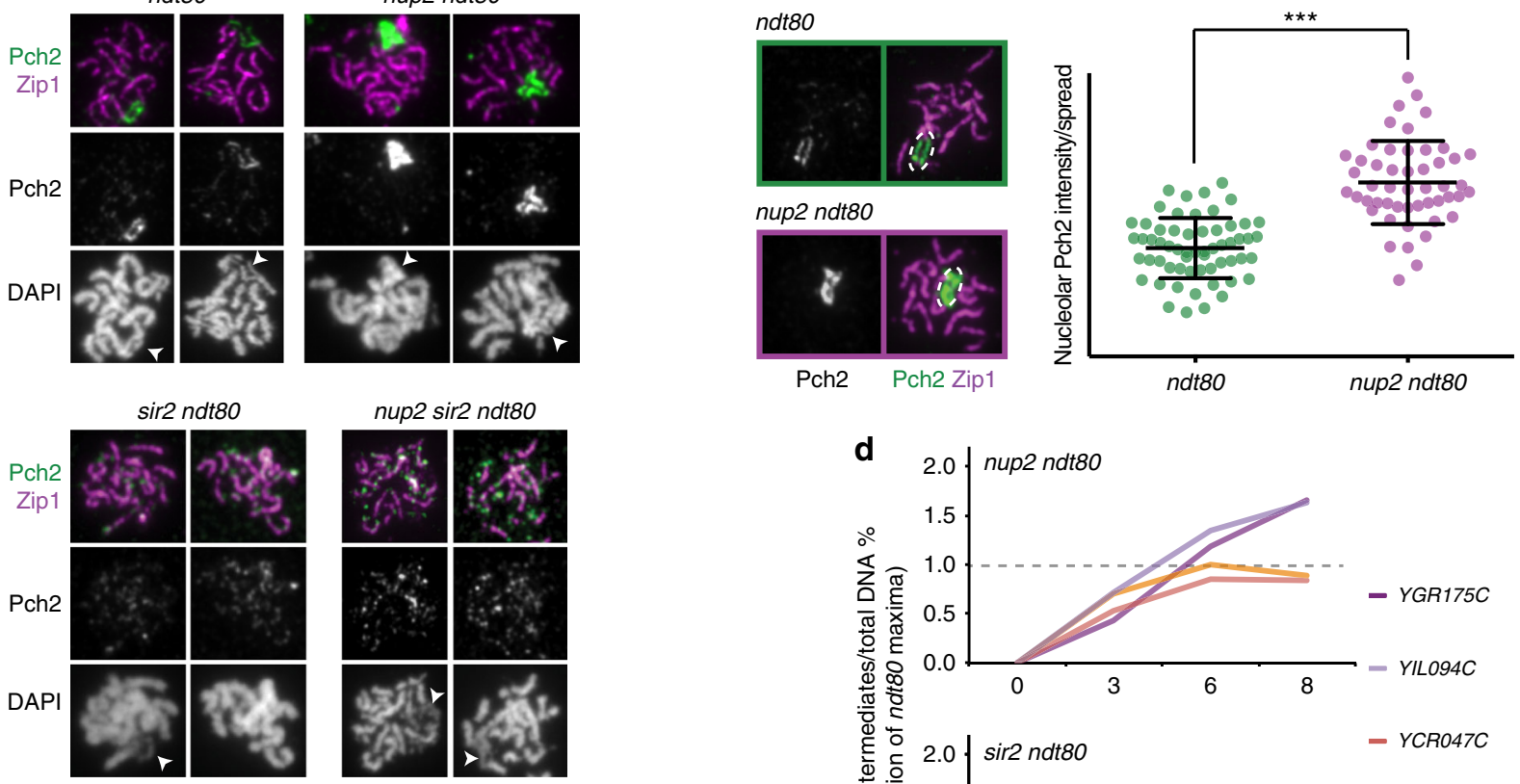

e
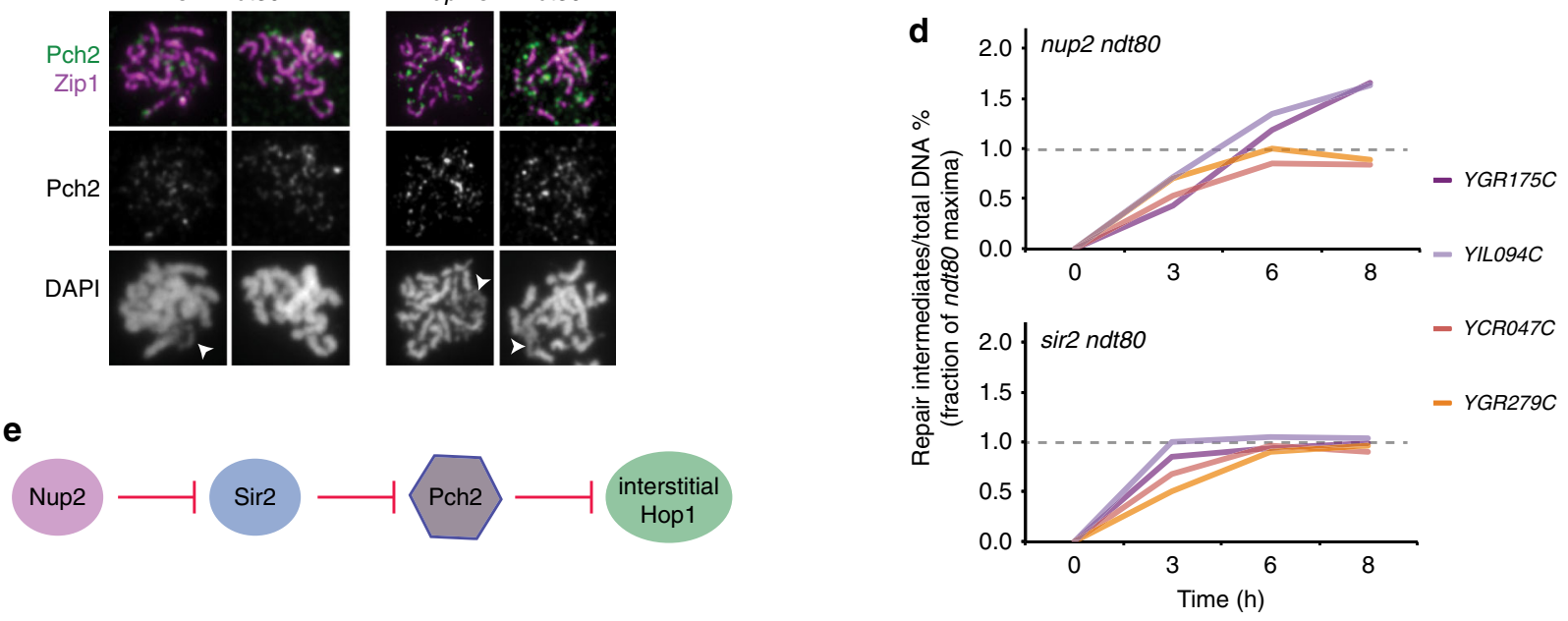

Fig. 7 Network of Nup2, Sir2, and Pch2 regulates Hop1 and DSBs on meiotic chromosomes. a Representative immunofluorescence images of Hop1 (green/

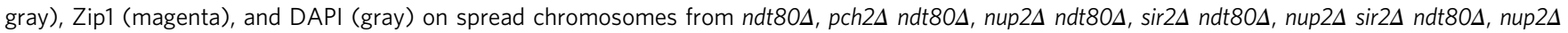

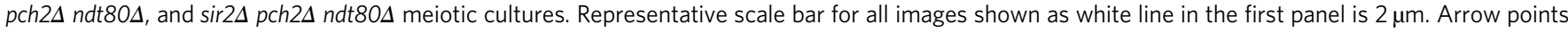
to a synapsed region (magenta), zoomed-in signals are shown in the bottom row and presence of overlap between Hop1 and Zip1 is indicated. $n>40$ for

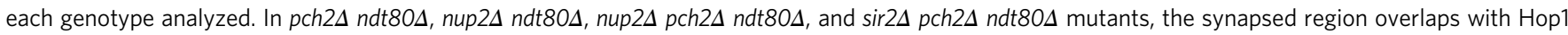
(green) but not in sir2 $\Delta$ nt $80 \Delta$, and nup $2 \Delta$ sir2 $\Delta$ ndt80 $\Delta$ mutant samples. Arrowhead points to rDNA where easily distinguishable. b Immunofluorescence

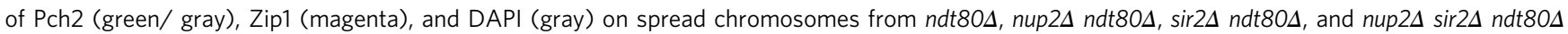
meiotic cultures. Arrowhead points to rDNA where distinguishable. c Right Panel: Quantification of nucleolar Pch2 intensity per spread nucleus in ndt80 $\Delta$ (green dots), and nup2 $\Delta$ ndt80 $\Delta$ (purple dots). $n>50$; error bars are S.D. from the mean. Left Panel: Representative immunofluorescence on a spread

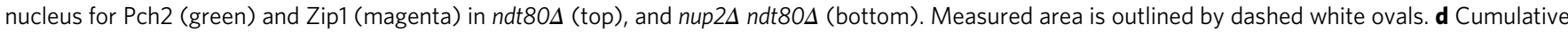

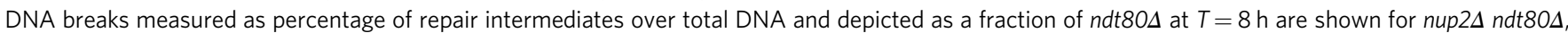
and sir2 $\Delta$ ndt80 $\Delta$ mutants (see Fig. 4e). Interstitial hotspots (YGR175C, YIL094C) are shown in shades of magenta and hotspots in EARs (YCR047C, YGR279C) are depicted in shades of orange. The data are averages of two independent biological replicates. e Schematic representation of genetic interaction between Sir2, Nup2, and Pch2 for evicting interstitial Hop1 from meiotic chromosomes 
a
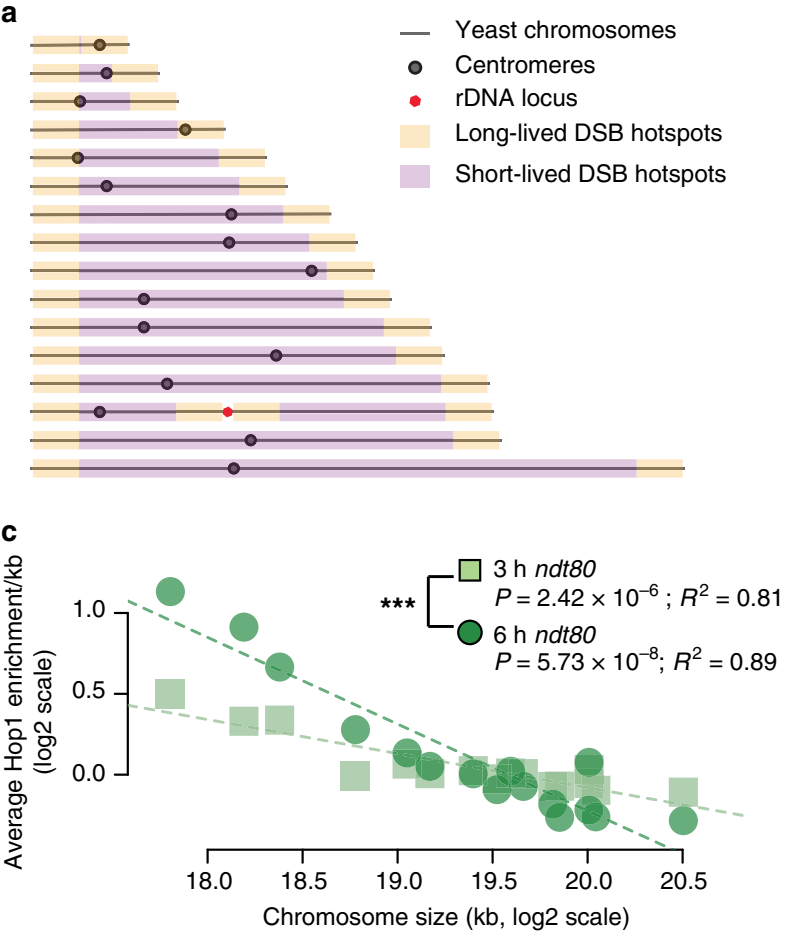

e

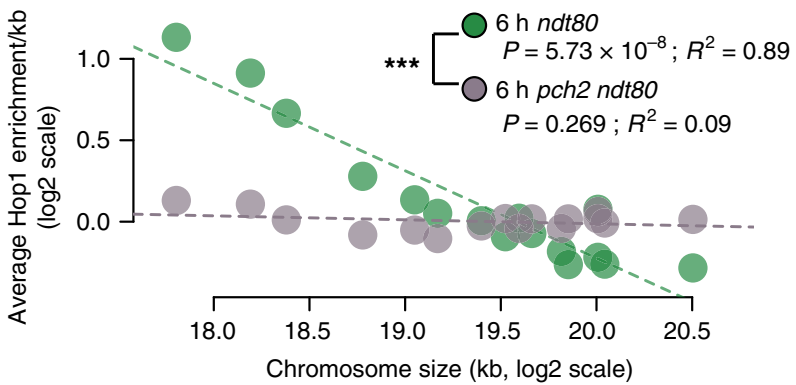

b
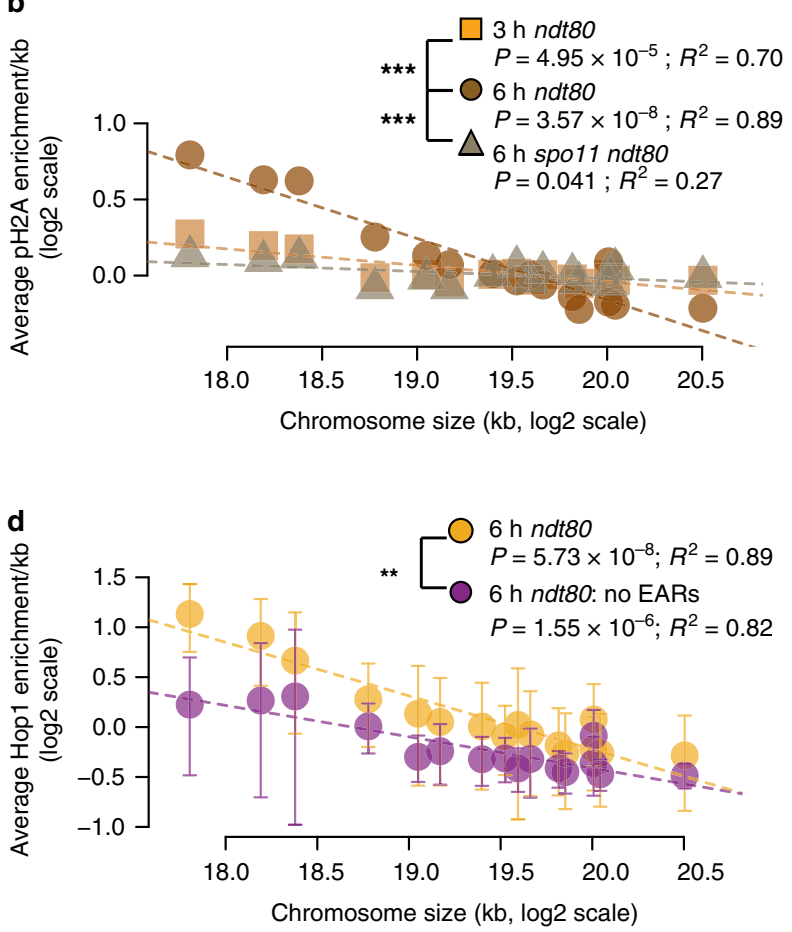

$\mathbf{f}$

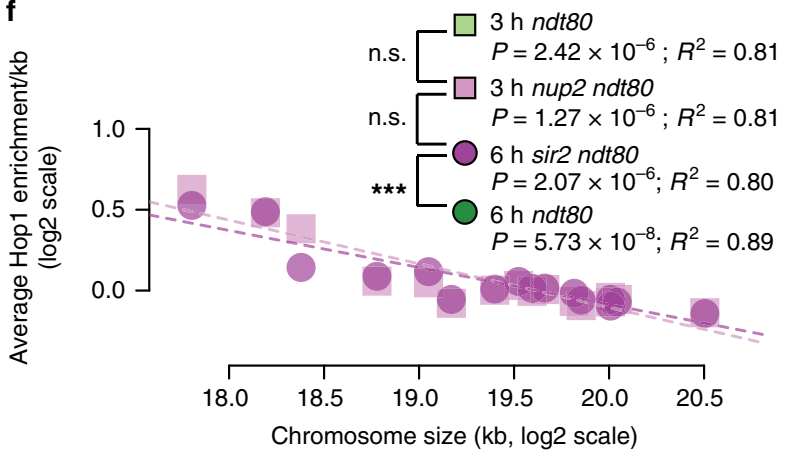

g

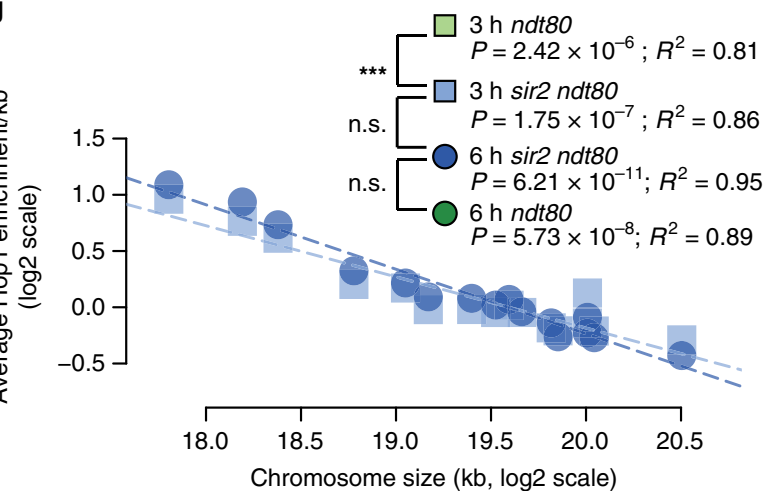

Fig. 8 Enrichment of Hop1 and DSB markers on short chromosomes increases significantly in late prophase. a Schematic of telomere-adjacent enrichment of Hop1 in late prophase predicts bias for DSBs on short chromosomes. The orange bars illustrate the large domains ( $100 \mathrm{~kb})$ of long-lived DSB hotspots at telomere-adjacent and rDNA-adjacent regions. Interstitial regions (magenta) harbor mainly the short-lived DSB hotspots. b-g Mean ChIP-seq enrichment per kb is plotted for each chromosome on log scale with regression analysis. $P$ and $R^{2}$ values are noted below the sample name. $R^{2}$, measure of the fit of the points to the line, can vary from 0 to 1.0 with 1.0 indicating a perfect fit. $P$ is the probability of obtaining large $R^{2}$ values. Two-way ANOVA was performed to test significant difference in the slope between the regression lines for different ChIP-seq samples. ANOVA-derived $P$ values are indicated, ${ }^{\star \star \star} P<0.001$. $\gamma$ - $\mathrm{H} 2 \mathrm{~A}$ ChIP-seq enrichment in early $(T=3 \mathrm{~h})$ and late prophase $(T=6 \mathrm{~h})$ in ndt $80 \Delta$ and late prophase $(T=6 \mathrm{~h})$ in spo11 $\mathrm{ndt} 80 \Delta$ in $\mathbf{b}$. Hop $1 \mathrm{ChIP}$-seq in early $(T=3 \mathrm{~h})$

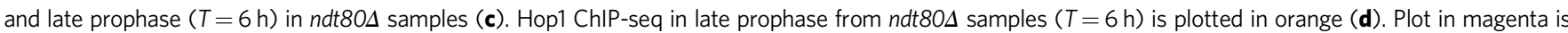
Hop1 enrichment from telomere-distal regions lacking EARs (110 kb from each chromosome end). Error bars are standard deviation of the means of 10 equal

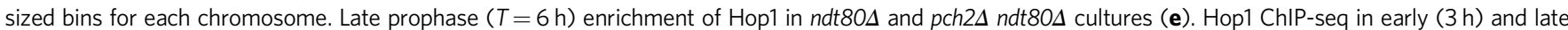

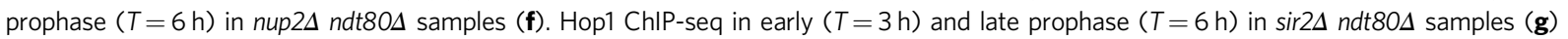


chromosome size bias in late prophase in both $n d t 80 \Delta$-arrested and wild-type cells (Fig. 8d, Supplementary Fig. 8d). These analyses indicate that EARs are at least partially responsible for the late prophase enrichment of Hopl on short chromosomes.

If EARs drive biased enrichment of Hopl on short chromosomes then this effect should be abrogated in pch $2 \Delta$ mutants, which do not exhibit Hop1 enrichment in EARs. Indeed, whereas the pattern of Hop1 chromosome size bias was not significantly different between $p c h 2 \triangle$ and $P C H 2$ samples in early prophase (ANOVA, $P=0.691$ ) (Supplementary Fig. 8e), in late prophase all chromosomes in pch $2 \Delta n d t 80 \Delta$ mutants had a similar Hop1 enrichment per $\mathrm{kb}$ irrespective of chromosome size (Fig. 8e). Hop1 chromosome size bias was also diminished in nup2 $\Delta$ mutants (Fig. 8f) but enhanced in sir2 $\Delta$ mutants (Fig. 8g). These observations indicate that a regulatory mechanism comprised of Nup2, Sir2, and Pch2 is responsible for maintaining the chromosome-size bias in meiotic prophase.

\section{Discussion}

Our findings uncover striking regional control of DSB potential during meiosis. DSB hotspots in large domains $(\sim 100 \mathrm{~kb})$ adjacent to chromosome ends, as well as regions bordering the rDNA locus, continue to break well after the SC down-regulates hotspots in interstitial chromosomal regions. This positional regulation increases the break potential on short chromosomes in the course of prophase and reveals a mechanism that promotes formation of the obligatory crossover on short chromosomes without having to measure chromosome length.

Research in several organisms, including yeast and mice, has strongly implicated the SC in mediating DSB down-regulation through the removal of HORMAD family proteins ${ }^{5,16,23,24}$. The results reported here are fully consistent with this view but show that, at least in yeast, chromosomes have specialized domains (EARs) that escape this down-regulation. A similar escape in regulation is also observed near the rDNA, consistent with previous observations ${ }^{16}$. Intriguingly, EARs retain Hop1 despite normal accumulation of the SC protein Zip1. Thus, although chromosome synapsis is spatially correlated with the removal of HORMAD family proteins ${ }^{23,24,27,28}$, Zip1 deposition in itself is clearly not sufficient for Hop1 eviction. In C. elegans, which also utilizes the SC to down-regulate DSB formation in a timely manner, $\mathrm{CO}$ designation is thought to lead to structural changes within the SC that prevent further DSB formation ${ }^{57,58}$. Similar structural changes may also occur in the yeast SC and may be required for Hop1 removal ${ }^{23}$. If so, then Hop1 in EARs is likely protected from these effects, either because Pch2-dependent removal is suppressed or because Hop 1 continues to load in these regions. The unique nature of EARs in this respect is highlighted by the fact that deletion of $\mathrm{PCH} 2$ leads to a build-up of Hop1 only in the interstitial regions, while EARs become comparatively under-enriched. These data suggest that Hop 1 binding in EARs is controlled by mechanisms distinct from the rest of the genome.

The molecular features that distinguish EARs from interstitial chromosomal regions remain to be discovered, although the consistent distance of EARs from chromosome ends points to a role for telomere-associated processes or the nuclear periphery. The latter possibility is particularly appealing because EAR-like domains of Hopl enrichment are also observed near the rDNA, which is located near the nuclear envelope. We did observe effects of NDJ1 and NUP2 deletion on overall Hop1 enrichments patterns but our data suggest that these effects are secondary to a failure to properly synapse or recruit Pch2 activity to the interstitial regions, respectively. Analysis of a limited set of additional telomeric regulators (TEL1, SIR3) and a nuclear envelope factor (ESC1) did not yield regulators of EAR establishment. These results obviously do not exclude redundant mechanisms or a role for different telomeric or nuclear-envelope factors. However, it is equally possible that other dynamic chromatin features, such as differences in replication timing, gene activity, or chromatin topology govern the Hop1 enrichment and provide the architectural basis of EARs.

We also observed unexpected dynamics of Hop1 around centromeres. Most notably, we found a strong centromeric enrichment of Hop1 in the earliest stages of prophase, before Hop1 has fully accumulated on chromosome arms. Centromeric Hop1 enrichment may similarly reflect nuclear architecture because prior to the tethering of telomeres to the nuclear envelope, the centromeres are clustered at the spindle pole body embedded in the nuclear envelope ${ }^{59}$. Interestingly, Spo11 is also enriched near centromeres before distributing to the arms ${ }^{45}$ and is likely active in these regions because we observe centromeric DSBs in early prophase (see Fig. 5c, left panel). Curiously, Mek1 is also enriched around centromeres. Centromeric recruitment of Mek1 is unexpected because Mek1 suppresses repair with the sister chromatid $^{23,48,60}$, yet DSBs at centromeres are thought to be channeled by Zip1 to primarily use the sister for recombination to protect against chromosome missegregation ${ }^{61,62}$. Perhaps, Zip1 suppresses Mek1 activity at the centromeres without evicting it. Another unexpected finding is that Pch2 suppresses DSBs around centromeres in late prophase. However, as COs are not enhanced around the centromeres in $p c h 2 \Delta$ mutants $^{63}$, Zip1 activity must be sufficient to prevent any deleterious interhomologue COs in this situation. These findings indicate that several mechanistic layers restrict DSBs and COs at centromeres, highlighting the importance of limiting COs in these regions.

Our findings also offer insights into the regulation of Pch2. We show that the nucleoporin Nup2 promotes the binding of Pch2 to synapsed chromosomes, and we provide genetic evidence that Nup2 functions by counteracting the histone deacetylase Sir2. This regulation may not be direct as Nup2 is a component of the nuclear pores, whereas Sir2 and Pch2 are strongly enriched in the nucleolus. We note, however, that Sir2 is also present at euchromatic replication origins ${ }^{64}$, which may also be sites of Pch2 activity $^{50}$. Furthermore, Nup2 is a mobile nucleoporin that interacts with chromatin to regulate transcription and contributes to boundary activity 65,66 , which may allow for interactions with Sir2 and the regulation of Hop1 on chromosomes.

Our analysis sheds important light on the mechanistic basis of the meiotic chromosome-size bias for recombination. In several organisms, including humans, short chromosomes exhibit higher levels of recombination ${ }^{32-34,63,67}$, a bias that in yeast is already apparent from elevated levels of axis protein deposition and DSB formation ${ }^{16,35-38}$. As EARs are of similar length regardless of chromosome size, EARs comprise a proportionally much larger fraction of short chromosomes (Fig. 8a). The bias in Hop1 enrichment could thus mediate the establishment of chromosome size bias in DSBs and COs.

Available data suggests that chromosome size bias for Hop1 enrichment in late prophase is a direct consequence of preferential SC-dependent removal of Hop1 from the interstitial regions. Consistent with this notion, disrupting either COassociated SC assembly (by deleting the CO-implementing factor ZIP3) or preventing the SC from removing Hop1 (by deleting $\mathrm{PCH} 2$ ) leads to a loss of chromosome size bias for recombination $16,63,68$. Intriguingly, in both situations, the failure to remove Hop1 differentially affects the EARs. In zip3 $\Delta$ mutants, DSB enrichment in EARs is diminished compared to wild type $(P=0.58$, Mann-Whitney-Wilcoxon test; Supplementary Fig. 8f). Similarly, the percentage of COs and noncrossovers (NCOs) per meiosis drops significantly in the EARs of pch $2 \Delta$ mutants, while average $\mathrm{CO}$ (and NCO) counts per chromosome surge with 
increasing chromosome size ${ }^{63}$. The persistence of Hop1 and Mek1 in the EARs likely also differentially affects repair progression $^{23}$. This regulation could explain the apparent increase in resection tract lengths observed on short chromosomes in late prophase ${ }^{43}$. Although short chromosomes in yeast are slowest to synapse $^{69}$, we find no evidence of reduced Zip1 accumulation on short chromosomes during the extended prophase of $n d t 80 \Delta$ cells (Supplementary Fig. 8g). These data suggest that synaptic delays are too small to be detected by ChIP-seq analysis and therefore cannot explain the elevated Hop1 levels on short chromosomes observed by the same assay.

COs are enriched in sub-telomeric regions in several organisms $s^{32,70-74}$. Recent work indicated that sub-telomeric regions in barley are the first to initiate recombination, leading to a relative depletion of recombination events in the interstitial regions ${ }^{75}$. This temporal pattern in recombination competence is opposite to the pattern reported here for S. cerevisiae, but the ultimate outcome of increased subtelomeric recombination is similar. Available data in yeast and humans is consistent with a telomere-guided effect in establishing these regions. Chromosome bisection caused a local increase in DSB levels in yeast ${ }^{35}$. Moreover, an ancient telomeric fusion that gave rise to human chromosome 2 led to a decrease in crossovers rates near the fused chromosome ends compared to chimpanzees, which maintained the two separate chromosomes ${ }^{70}$. Thus, some features of EARs may well be evolutionarily conserved.

We propose that EARs provide a safety mechanism that ensures that DSB formation is not prematurely inactivated by the formation of the SC. Premature down-regulation of the DSB machinery is particularly problematic for short chromosomes because of their inherently lower number of DSB hotspots. By establishing privileged regions that are refractory to this downregulation, cells may ensure that all chromosomes retain a (limited) potential for DSB formation and successful crossover recombination throughout meiotic prophase.

\section{Methods \\ Method details. Growth conditions: Synchronous meiotic time-courses were performed in the following manner ${ }^{23}$. The strains were first patched on glycerol media (YPG) and then transferred to rich media with $4 \%$ dextrose (YPD $4 \%$ ). The cells were then grown at $23^{\circ} \mathrm{C}$ for $24 \mathrm{~h}$ in liquid YPD and diluted into pre- sporulation media (BYTA, $1 \%$ yeast extract, $2 \%$ bactopeptone, $1 \%$ potassium acetate, $50 \mathrm{mM}$ potassium phthalate) at $\mathrm{A}_{600} 0.3$. The BYTA culture was grown at $30{ }^{\circ} \mathrm{C}$ for $16 \mathrm{~h}$. The cells were washed twice in sterile water and transferred to sporulation media $\left(0.3 \%\right.$ potassium acetate) at $30^{\circ} \mathrm{C}$ to induce synchronous sporulation. Samples for ChIP-seq $(25 \mathrm{~mL})$ or DSB Southern assays $(10 \mathrm{~mL})$ were collected at the indicated timepoints. Growth conditions for obtaining Spo11-oligo sequences were as follows ${ }^{44}$. Cells were grown at $30^{\circ} \mathrm{C}$ in $1 \%$ yeast extract, $2 \%$ bactopeptone, $1 \%$ potassium acetate for $14 \mathrm{~h}$ and sporulated in $2 \%$ potassium acetate.}

Experimental model and subject details. All strains used in this study are listed in Supplementary Table 1 and are in the SK1 background except where noted.

DSB Southern analysis. Meiotic cells collected at the indicated time points were embedded in agarose plugs to minimize background from random shearing and genomic DNA was extracted ${ }^{50}$. The plugs were washed $4 \times$ in TE (Tris/EDTA) for $1 \mathrm{~h}$ each wash followed by $4 \times 1 \mathrm{~h}$ washes in the appropriate NEB buffer (New England Biolabs). Plugs for each time-point were transferred to separate tubes and melted at $65^{\circ} \mathrm{C}$. The genomic DNA in molten agarose was equilibrated at 42 ${ }^{\circ} \mathrm{C}$ prior to incubation with appropriate restriction enzyme(s). The digested DNA was electrophoresed in $0.8 \%$ agarose (Seakem LE) in $1 \times$ TBE (Tris/Borate/ EDTA) at $2.6 \mathrm{~V} \mathrm{~cm}^{-1}$ for $18 \mathrm{~h}$. The DNA was transferred to a Hybond-XL nylon membrane (GE Healthcare) by capillary transfer and detected by Southern hybridization ${ }^{23}$. Restriction enzymes used to fragment genomic DNA for DSB analysis, as well as the primer sequences to construct probes are listed in the Supplementary Table 2 . Probes labeled with ${ }^{32} \mathrm{P}$ dCTP were generated using the listed primers and a Prime-It random labeling kit (Agilent, catalog\# 300392). Southern blots were exposed on an Fuji imaging screen and the phospho-signal was detected on Typhoon FLA 9000 (GE) and quantified using Image software 'http://imagej.nih.gov/ij/'. In ndt80A samples, repair intermediates provide a cumulative view of all DSB occurrence. Thus, in Southerns where DSB signals were faint (Supplementary Fig. 5b), the sum of DSBs and repair intermediates was quantified. Only repair intermediates were quantified in Figs 4e, 7d, because DSB signals were very faint and could not be quantified reliably. Plots were generated using the Graphpad program in Prism.

Mechanical spreading of chromosomes and immunofluorescence. Meiotic cells collected at indicated timepoints were treated with $200 \mathrm{mM}$ Tris pH7.5/20 mM dithiothreitol (DTT) for $2 \mathrm{~min}$ at room temperature. Cells were spheroplasted at $30{ }^{\circ} \mathrm{C}$ in $2 \%$ potassium acetate $/ 1 \mathrm{M}$ sorbitol $/ 0.13 \mu \mathrm{g} / \mu \mathrm{L}$ zymolyase $\mathrm{T} 100$. The spheroplasts were gently washed and resuspended in ice-cold 0.1 M MES pH6.4/1 $\mathrm{mM}$ EDTA/0.5 mM $\mathrm{MgCl}_{2} / 1 \mathrm{M}$ sorbitol. Two volumes of $3 \%$ para-formaldehyde/ $3.4 \%$ sucrose was added to spheroplasts on clean glass slide (soaked in ethanol and air-dried) immediately followed by four volumes of $1 \%$ Lipsol. The contents were mixed by gently tilting the slide. After at least $1 \mathrm{~min}$, four volumes of $3 \%$ paraformaldehyde $/ 3.4 \%$ sucrose was added to the samples. The chromosome spreads were then made by mechanically spreading the samples with a glass rod. The samples were rinsed in $0.4 \%$ Photoflo (Kodak, catalog\# 1464510), dried overnight at room temperature and stored at $-80^{\circ} \mathrm{C}$.

Immunofluorescence on the slides was performed with the desired primary antibodies followed by fluorescent-conjugated secondary antibodies listed in Supplementary Table 3. Deltavision Elite imaging system (GE) equipped with Olympus $\times 100$ lens/1.40 NA UPLSAPO PSF oil immersion lens and an InsightSSI Solid State Illumination module was used to image samples. Images were captured using the Evolve 512 EMCCD camera in the conventional mode and analyzed with softWoRx 7.0.0 software. SoftWoRx 7.0.0 software was also used to quantify signal. DAPI was used to identify the chromosomal signal. An oval was created around the nucleolar Pch2 signal (which is more intense than nuclear Pch2) to quantify nucleolar Pch2 in Fig. 7c. Scatterplots were generated using Graphpad program in Prism and statistical significance was determined using Mann-Whitney-Wilcoxon test.

Chromatin immunoprecipitation and Illumina sequencing. Twenty-five milliliter of sample was collected from sporulation cultures at the indicated time points and crosslinked in $1 \%$ formaldehyde (Sigma) for $30 \mathrm{~min}$. Formaldehyde was quenched with $125 \mathrm{mM}$ glycine. ChIP was performed by lysing the cells in $500 \mu \mathrm{l}$ of lysis buffer $(50 \mathrm{mM}$ HEPES/KOH pH 7.5, $140 \mathrm{mM} \mathrm{NaCl}, 1 \mathrm{mM}$ EDTA, $1 \%$ Triton X$100,0.1 \%$ sodium deoxycholate) with protease inhibitors (1 mM PMSF, $1 \mathrm{mM}$ Benzamidine, $1 \mathrm{mg} \mathrm{ml}^{-1}$ Bacitracin, one Roche Tablet (catalog\# 11836170001) in $10 \mathrm{ml}$ ) and glass beads in a biopulveriser ${ }^{76}$. The sample was sonnicated to obtain DNA at an average length of $500 \mathrm{bp}$. Immunoprecipitation was performed on clarified sample using antibodies listed in the Supplementary Table 3. Libraries for ChIP sequencing were prepared by PCR amplification using Illumina TruSeq DNA sample preparation kits $\mathrm{v} 1$ and $\mathrm{v} 2$ but adaptors were used at 1:20 dilution ${ }^{10}$. Quality of the libraries was checked on 2100 Bioanalyzer or 2200 Tapestation. Libraries were quantified using qPCR prior to pooling. The ChIP libraries were sequenced on Illumina HiSeq 2500 or NextSeq 500 instruments at the NYU Biology Genomics core to yield 51/50 bp single-end reads. For spike-in normalization (SNP-ChIP), SK288c crosslinked meiotic cells were added to respective samples at $10-20 \%$ prior to ChIP processing ${ }^{46}$. SNP-ChIP libraries were sequenced on NextSeq 500 to yield 100 bp single-end reads. All ChIP-seq data sets are listed in Supplementary Table 4.

Processing of reads from Illumina sequencing. Illumina output reads were processed in the following manner ${ }^{77}$. The reads were mapped to SK1 genome ${ }^{78}$ using Bowtie $^{79}$. SNP-ChIP library reads were aligned to concatenated genome assemblies of SK1 and S288c genomes ${ }^{46}$. Only reads that mapped to a single position and also matched perfectly to the reference genome were retrieved for further analysis. 3' ends of the reads were extended to a final length of $200 \mathrm{bp}$ using MACS2 2.1.1 'https://github.com/taoliu/MACS' and probabilistically determined PCR duplicates were removed. The input and ChIP pileups were SPMRnormalized (signal per million reads) and fold-enrichment of ChIP over input data was used for further analyses. The pipeline used to process Illumina reads can be found at 'https:/github.com/hochwagenlab/ChIPseq_functions/tree/master/ ChIPseq_Pipeline_v3/'. The pipeline used to process SNP-ChIP reads and calculate spike-in normalization factor can be found at 'https://github.com/hochwagenlab/ ChIPseq_functions/tree/master/ChIPseq_Pipeline_hybrid_genome/ '.

Spo11-oligo mapping. Spo11-oligos were immunoprecipitated from $50 \mathrm{ml}$ of synchronous meiotic culture ( $T=4 \mathrm{~h}$ samples) with $80 \mu \mathrm{l}$ of the anti-FLAG antibody in the first round and $25 \mu$ in the second round of immunoprecipitation ${ }^{44}$ The Spo11-oligos were sequenced on Illumina HiSeq in the Memorial Sloan Kettering Cancer Center (MSKCC) Integrated Genomics Operation core facility. The adaptors were clipped followed by alignment of the oligo reads to S288c (sacCer2) reference genome using a custom pipeline ${ }^{16,38}$. Averaged maps from biological replicates were used for further analysis. Oligos within the rDNA (coordinates 451,000 and 471,000 on ChrXII) are highly enriched in sir2 $\Delta$ datasets and were removed from all datasets prior to analysis. Spo11-oligo datasets analyzed in this manuscript are listed in Supplementary Table 5. 
Quantification and statistical analyses. ChIP-seq data from two biological replicates were merged prior to analyses using the ChIPseq_Pipeline_v3 except for

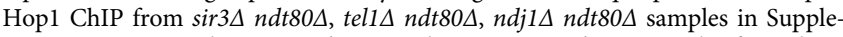
mentary Fig. $7 \mathrm{a}$ and No tag $n d t 80 \Delta$, and NDJ1-FRB ndt80 $\Delta$ samples for Ndj1 depletion studies in Supplementary Fig. 7b for which replicate experiments were not performed. All datasets were normalized to global mean of one and regional enrichment was calculated. The R functions used can be found at 'https://github. com/hochwagenlab/hwglabr2/' and 'https://github.com/VijiSubramanian/chrEnds'.

Statistical significance tests were performed in R 3.3.3. Either Mann-WhitneyWilcoxon test on mean enrichment by chromosome (16 chromosomes; EARs versus interstitial) was used to test for significance or one-way ANOVA with posthoc Tukey test was used. Two-way ANOVA for multiple linear regressions with interaction was performed on log2-scaled ChIP-seq enrichment to test variation in the slopes (chromosome size bias) of two different samples. For bootstrap analyses, random samplings of the ChIP data were performed on each of the 16 circularized chromosomes and this was repeated 5000 times. The samplings were equivalent to the experimental query in size and number for each experiment. Specifically, bootstrap samplings for EARs were two unlinked samplings from each of the 16 chromosomes, for the centromeres bootstrap involved only a single sampling of each of the 16 chromosomes while for the rDNA bootstrap the entire genome was sampled twice. Additionally, to assay enrichment at the rDNA borders, EARs (120 $\mathrm{kb}$ from either telomere) were excluded from the genome for random bootstrap samplings. Both averaged random sampling data and experimental query were normalized to genome average. The median and two-sided 95\% CI was calculated based on the spread of the bootstrap-derived distribution of enrichment.

Reporting summary. Further information on experimental design is available in the Nature Research Reporting Summary linked to this article.

\section{Data availability}

The source data underlying all Figures and Supplementary Figures are provided as a Source Data file. All datasets reported in this paper (except published datasets) are available at the Gene Expression Omnibus (GEO) with the accession number 'GSE105111' for ChIP-seq datasets and 'GSE122882' for Spo11-oligo datasets. All data is available from the authors upon reasonable request. Genome-wide S1-seq datasets (Supplementary Table 6) for wild-type meiosis, GEO accession number GSE85253, were obtained from ref. ${ }^{43}$. The processed dataset aligned to the $\mathrm{S} 288 \mathrm{c}$ reference genome (sacCer2) was used. The mapped Spol1-oligo counts within hotspots for wild type and zip3 3 mutant, GEO number GSE48299, aligned to the S288c reference genome (sacCer2) were obtained from ref. ${ }^{16}$. Wild type datasets from GEO dataset GSE67910 ${ }^{44}$ were used as control for Spo11-Flag oligo sequencing data from $p c h 2 \Delta$ and $\operatorname{sir} 2 \Delta$ samples.

Received: 5 September 2018 Accepted: 5 February 2019

Published online: 27 February 2019

\section{References}

1. de Massy, B. Initiation of meiotic recombination: how and where? Conservation and specificities among eukaryotes. Annu. Rev. Genet. 47, 563-99 (2013).

2. Lam, I. \& Keeney, S. Mechanism and regulation of meiotic recombination initiation. Cold Spring Harb. Perspect. Biol. 7, a016634 (2014).

3. Cooper, T. J., Garcia, V. \& Neale, M. J. Meiotic DSB patterning: a multifaceted process. Cell Cycle 15, 13-21 (2016).

4. Subramanian, V. V. \& Hochwagen, A. The meiotic checkpoint network: stepby-step through meiotic prophase. Cold Spring Harb. Perspect. Biol. 6, a016675 (2014).

5. Keeney, S., Lange, J. \& Mohibullah, N. Self-organization of meiotic recombination initiation: general principles and molecular pathways. Annu. Rev. Genet. 48, 187-214 (2014)

6. Blat, Y., Protacio, R. U., Hunter, N. \& Kleckner, N. Physical and functional interactions among basic chromosome organizational features govern early steps of meiotic chiasma formation. Cell 111, 791-802 (2002).

7. Panizza, S. et al. Spol1-accessory proteins link double-strand break sites to the chromosome axis in early meiotic recombination. Cell 146, 372-83 (2011).

8. Mao-Draayer, Y., Galbraith, A. M., Pittman, D. L., Cool, M. \& Malone, R. E. Analysis of meiotic recombination pathways in the yeast Saccharomyces cerevisiae. Genetics 144, 71-86 (1996).

9. Xu, L., Weiner, B. M. \& Kleckner, N. Meiotic cells monitor the status of the interhomolog recombination complex. Genes Dev. 11, 106-18 (1997).

10. Sun, $\mathrm{X}$. et al. Transcription dynamically patterns the meiotic chromosomeaxis interface. eLife 4, e07424 (2015).

11. Allers, T. \& Lichten, M. Differential timing and control of noncrossover and crossover recombination during meiosis. Cell 106, 47-57 (2001).
12. Argunhan, B. et al. Direct and indirect control of the initiation of meiotic recombination by DNA damage checkpoint mechanisms in budding yeast. PLoS ONE 8, e65875 (2013)

13. Blitzblau, H. G. \& Hochwagen, A. ATR/Mec1 prevents lethal meiotic recombination initiation on partially replicated chromosomes in budding yeast. eLife 2, e00844 (2013).

14. Gray, S., Allison, R. M., Garcia, V., Goldman, A. S. \& Neale, M. J. Positive regulation of meiotic DNA double-strand break formation by activation of the DNA damage checkpoint kinase Mec1(ATR). Open Biol. 3, 130019 (2013).

15. Murakami, H. \& Keeney, S. Temporospatial coordination of meiotic DNA replication and recombination via DDK recruitment to replisomes. Cell 158, 861-873 (2014).

16. Thacker, D., Mohibullah, N., Zhu, X. \& Keeney, S. Homologue engagement controls meiotic DNA break number and distribution. Nature 510, 241-6 (2014).

17. Tonami, Y., Murakami, H., Shirahige, K. \& Nakanishi, M. A checkpoint control linking meiotic $\mathrm{S}$ phase and recombination initiation in fission yeast. Proc. Natl Acad. Sci. USA 102, 5797-801 (2005).

18. Carballo, J. A. et al. Budding yeast ATM/ATR control meiotic double-strand break (DSB) levels by down-regulating Rec114, an essential component of the DSB-machinery. PLoS Genet. 9, e1003545 (2013).

19. Garcia, V., Gray, S., Allison, R. M., Cooper, T. J. \& Neale, M. J. Tel1(ATM)mediated interference suppresses clustered meiotic double-strand-break formation. Nature 520, 114-8 (2015).

20. Mohibullah, N. \& Keeney, S. Numerical and spatial patterning of yeast meiotic DNA breaks by Tel1. Genome Res. 27, 278-288 (2017).

21. Zhang, L., Kim, K. P., Kleckner, N. E. \& Storlazzi, A. Meiotic double-strand breaks occur once per pair of (sister) chromatids and, via Mec1/ATR and Tel1/ATM, once per quartet of chromatids. Proc. Natl Acad. Sci. USA 108, 20036-41 (2011).

22. Kauppi, L. et al. Numerical constraints and feedback control of double-strand breaks in mouse meiosis. Genes Dev. 27, 873-86 (2013).

23. Subramanian, V. V. et al. Chromosome synapsis alleviates Mek1-dependent suppression of meiotic DNA repair. PLoS Biol. 14, e1002369 (2016).

24. Wojtasz, L. et al. Mouse HORMAD1 and HORMAD2, two conserved meiotic chromosomal proteins, are depleted from synapsed chromosome axes with the help of TRIP13 AAA-ATPase. PLoS Genet. 5, e1000702 (2009).

25. Rosu, S. et al. The C. elegans DSB-2 protein reveals a regulatory network that controls competence for meiotic DSB formation and promotes crossover assurance. PLoS Genet. 9, el003674 (2013).

26. Stamper, E. L. et al. Identification of DSB-1, a protein required for initiation of meiotic recombination in Caenorhabditis elegans, illuminates a crossover assurance checkpoint. PLoS Genet. 9, e1003679 (2013).

27. Borner, G. V., Barot, A. \& Kleckner, N. Yeast Pch2 promotes domainal axis organization, timely recombination progression, and arrest of defective recombinosomes during meiosis. Proc. Natl Acad. Sci. USA 105, 3327-32 (2008).

28. San-Segundo, P. A. \& Roeder, G. S. Pch2 links chromatin silencing to meiotic checkpoint control. Cell 97, 313-24 (1999).

29. Herruzo, E. et al. The Pch2 AAA + ATPase promotes phosphorylation of the Hop1 meiotic checkpoint adaptor in response to synaptonemal complex defects. Nucleic Acids Res. 44, 7722-41 (2016).

30. Roig, I. et al. Mouse TRIP13/PCH2 is required for recombination and normal higher-order chromosome structure during meiosis. PLoS Genet. 6, e1001062 (2010).

31. Xu, L., Ajimura, M., Padmore, R., Klein, C. \& Kleckner, N. NDT80, a meiosisspecific gene required for exit from pachytene in Saccharomyces cerevisiae. Mol. Cell. Biol. 15, 6572-81 (1995).

32. Backstrom, N. et al. The recombination landscape of the zebra finch Taeniopygia guttata genome. Genome Res. 20, 485-95 (2010).

33. Kaback, D. B. Chromosome-size dependent control of meiotic recombination in humans. Nat. Genet. 13, 20-1 (1996).

34. Kaback, D. B., Guacci, V., Barber, D. \& Mahon, J. W. Chromosome sizedependent control of meiotic recombination. Science 256, 228-32 (1992).

35. Blitzblau, H. G., Bell, G. W., Rodriguez, J., Bell, S. P. \& Hochwagen, A. Mapping of meiotic single-stranded DNA reveals double-stranded-break hotspots near centromeres and telomeres. Curr. Biol. 17, 2003-12 (2007).

36. Gerton, J. L. et al. Global mapping of meiotic recombination hotspots and coldspots in the yeast Saccharomyces cerevisiae. Proc. Natl Acad. Sci. USA 97, 11383-90 (2000).

37. Lam, I. \& Keeney, S. Nonparadoxical evolutionary stability of the recombination initiation landscape in yeast. Science 350, 932-7 (2015).

38. Pan, J. et al. A hierarchical combination of factors shapes the genome-wide topography of yeast meiotic recombination initiation. Cell 144, 719-31 (2011)

39. Chu, S. \& Herskowitz, I. Gametogenesis in yeast is regulated by a transcriptional cascade dependent on Ndt80. Mol. Cell 1, 685-96 (1998).

40. Shroff, R. et al. Distribution and dynamics of chromatin modification induced by a defined DNA double-strand break. Curr. Biol. 14, 1703-11 (2004). 
41. Unal, E. et al. DNA damage response pathway uses histone modification to assemble a double-strand break-specific cohesin domain. Mol. Cell 16, 991-1002 (2004)

42. Kim, J. A., Kruhlak, M., Dotiwala, F., Nussenzweig, A. \& Haber, J. E. Heterochromatin is refractory to gamma- $\mathrm{H} 2 \mathrm{AX}$ modification in yeast and mammals. J. Cell. Biol. 178, 209-18 (2007).

43. Mimitou, E. P., Yamada, S. \& Keeney, S. A global view of meiotic doublestrand break end resection. Science 355, 40-45 (2017).

44. Zhu, X. \& Keeney, S. High-resolution global analysis of the influences of Bas1 and Ino4 transcription factors on meiotic DNA break distributions in Saccharomyces cerevisiae. Genetics 201, 525-42 (2015).

45. Kugou, K. et al. Rec8 guides canonical Spo11 distribution along yeast meiotic chromosomes. Mol. Biol. Cell. 20, 3064-76 (2009).

46. Vale-Silva, L. A., Markowitz, T. E. \& Hochwagen, A. SNP-ChIP: A versatile and tag-free method to quantify changes in protein binding across the genome. BMC Genom. 20, 54 (2019).

47. Carballo, J. A., Johnson, A. L., Sedgwick, S. G. \& Cha, R. S. Phosphorylation of the axial element protein Hopl by Mecl/Tell ensures meiotic interhomolog recombination. Cell 132, 758-70 (2008).

48. Niu, H. et al. Partner choice during meiosis is regulated by Hop1-promoted dimerization of Mek1. Mol. Biol. Cell. 16, 5804-18 (2005).

49. Kniewel, R. et al. Histone $\mathrm{H} 3$ threonine 11 phosphorylation is catalyzed directly by the meiosis-specific kinase Mek1 and provides a molecular readout of Mek1 activity in vivo. Genetics 207, 1313-1333 (2017).

50. Vader, G. et al. Protection of repetitive DNA borders from self-induced meiotic instability. Nature 477, 115-9 (2011).

51. Smith, A. V. \& Roeder, G. S. The yeast Red1 protein localizes to the cores of meiotic chromosomes. J. Cell. Biol. 136, 957-67 (1997).

52. Ho, H. C. \& Burgess, S. M. Pch2 acts through Xrs2 and Tel1/ATM to modulate interhomolog bias and checkpoint function during meiosis. PLoS Genet. 7, e1002351 (2011).

53. Conrad, M. N., Dominguez, A. M. \& Dresser, M. E. Ndj1p, a meiotic telomere protein required for normal chromosome synapsis and segregation in yeast. Science 276, 1252-5 (1997).

54. Chu, D. B., Gromova, T., Newman, T. A. C. \& Burgess, S. M. The nucleoporin Nup2 contains a meiotic-autonomous region that promotes the dynamic chromosome events of meiosis. Genetics 206, 1319-1337 (2017).

55. Niu, H. et al. Mek1 kinase is regulated to suppress double-strand break repair between sister chromatids during budding yeast meiosis. Mol. Cell. Biol. 27, 5456-67 (2007).

56. Woltering, D. et al. Meiotic segregation, synapsis, and recombination checkpoint functions require physical interaction between the chromosomal proteins Red1p and Hop1p. Mol. Cell. Biol. 20, 6646-58 (2000).

57. Hayashi, M., Mlynarczyk-Evans, S. \& Villeneuve, A. M. The synaptonemal complex shapes the crossover landscape through cooperative assembly, crossover promotion and crossover inhibition during Caenorhabditis elegans meiosis. Genetics 186, 45-58 (2010).

58. Libuda, D. E., Uzawa, S., Meyer, B. J. \& Villeneuve, A. M. Meiotic chromosome structures constrain and respond to designation of crossover sites. Nature 502, 703-706 (2013).

59. Hayashi, A., Ogawa, H., Kohno, K., Gasser, S. M. \& Hiraoka, Y. Meiotic behaviours of chromosomes and microtubules in budding yeast: relocalization of centromeres and telomeres during meiotic prophase. Genes. Cells 3, 587-601 (1998).

60. Wu, H. Y., Ho, H. C. \& Burgess, S. M. Mek1 kinase governs outcomes of meiotic recombination and the checkpoint response. Curr. Biol. 20, 1707-16 (2010).

61. Chen, S. Y. et al. Global analysis of the meiotic crossover landscape. Dev. Cell. 15, 401-15 (2008)

62. Vincenten, N. et al. The kinetochore prevents centromere-proximal crossover recombination during meiosis. Elife 4, e10850 (2015)

63. Chakraborty, P. et al. Modulating crossover frequency and interference for obligate crossovers in Saccharomyces cerevisiae meiosis. G3 7, 1511-1524 (2017).

64. Hoggard, T. A. et al. Yeast heterochromatin regulators Sir2 and Sir3 act directly at euchromatic DNA replication origins. PLoS Genet. 14, e1007418 (2018).

65. Dilworth, D. J. et al. The mobile nucleoporin Nup2p and chromatin-bound Prp20p function in endogenous NPC-mediated transcriptional control. J. Cell. Biol. 171, 955-65 (2005).

66. Ishii, K., Arib, G., Lin, C., Van Houwe, G. \& Laemmli, U. K. Chromatin boundaries in budding yeast: the nuclear pore connection. Cell 109, 551-62 (2002).

67. Lange, J. et al. The landscape of mouse meiotic double-strand break formation, processing, and repair. Cell 167, 695-708 (2016).

68. Zanders, S., Sonntag Brown, M., Chen, C. \& Alani, E. Pch2 modulates chromatid partner choice during meiotic double-strand break repair in Saccharomyces cerevisiae. Genetics 188, 511-21 (2011).
69. Lee, C. Y., Conrad, M. N. \& Dresser, M. E. Meiotic chromosome pairing is promoted by telomere-led chromosome movements independent of bouquet formation. PLoS Genet. 8, e1002730 (2012).

70. Auton, A. et al. A fine-scale chimpanzee genetic map from population sequencing. Science 336, 193-8 (2012).

71. Barnes, T. M., Kohara, Y., Coulson, A. \& Hekimi, S. Meiotic recombination, noncoding DNA and genomic organization in Caenorhabditis elegans. Genetics 141, 159-79 (1995).

72. Barton, A. B., Pekosz, M. R., Kurvathi, R. S. \& Kaback, D. B. Meiotic recombination at the ends of chromosomes in Saccharomyces cerevisiae. Genetics 179, 1221-35 (2008).

73. Singhal, S. et al. Stable recombination hotspots in birds. Science 350, 928-32 (2015).

74. Yu, A. et al. Comparison of human genetic and sequence-based physical maps. Nature 409, 951-3 (2001).

75. Higgins, J. D. et al. Spatiotemporal asymmetry of the meiotic program underlies the predominantly distal distribution of meiotic crossovers in barley. Plant Cell 24, 4096-109 (2012).

76. Blitzblau, H. G., Chan, C. S., Hochwagen, A. \& Bell, S. P. Separation of DNA replication from the assembly of break-competent meiotic chromosomes. PLoS Genet. 8, e1002643 (2012).

77. Paul, M. R., Markowitz, T. E., Hochwagen, A. \& Ercan, S. Condensin depletion causes genome decompaction without altering the level of global gene expression in Saccharomyces cerevisiae. Genetics 210, 331-344 (2018).

78. Yue, J. X. et al. Contrasting evolutionary genome dynamics between domesticated and wild yeasts. Nat. Genet. 49, 913-924 (2017).

79. Langmead, B., Trapnell, C., Pop, M. \& Salzberg, S. L. Ultrafast and memoryefficient alignment of short DNA sequences to the human genome. Genome Biol. 10, R25 (2009).

\section{Acknowledgements}

We are grateful to Brian Parker at NYU for advice on statistics. We thank the NYU Genomics Core facility for technical assistance and data processing. This work was funded in part by grant R01 GM111715 from the NIH and research grant \#6-FY16-208 from the March of Dimes Foundation to A.H.; grant R35 GM118092 from the NIH to S.K. and MSKCC Cancer Center Core Grant P30 CA008748; grant R01 GM050717 from the NIH to N.M.H.; grants BFU2015-65417-R from MINECO and CSI084U16 from Junta de Castilla y León in Spain to P.A.S.

\section{Author contributions}

Conceptualization, V.V.S. and A.H.; Investigation, V.V.S., X.Z., S.K. and A.H.; Software, V.V.S., T.E.M., L.A.V.S. and A.H.; Formal analysis, V.V.S. and A.H.; Resources, V.V.S., P.A.S, N.M.H. and A.H.; Writing-original draft, V.V.S. and A.H.; Writing-review and editing, V.V.S., X.Z., T.E.M., L.A.V.S., P.A.S, N.M.H., S.K. and A.H.

\section{Additional information}

Supplementary Information accompanies this paper at https://doi.org/10.1038/s41467019-08875-x.

\section{Competing interests: The authors declare no competing interests.}

Reprints and permission information is available online at http://npg.nature.com/ reprintsandpermissions/

Journal peer review information: Nature Communications thanks Valerie Borde and the other anonymous reviewer(s) for their contribution to the peer review of this work. Peer reviewer reports are available.

Publisher's note: Springer Nature remains neutral with regard to jurisdictional claims in published maps and institutional affiliations.

\footnotetext{
Open Access This article is licensed under a Creative Commons BY Attribution 4.0 International License, which permits use, sharing
adaptation, distribution and reproduction in any medium or format, as long as you give appropriate credit to the original author(s) and the source, provide a link to the Creative Commons license, and indicate if changes were made. The images or other third party material in this article are included in the article's Creative Commons license, unless indicated otherwise in a credit line to the material. If material is not included in the article's Creative Commons license and your intended use is not permitted by statutory regulation or exceeds the permitted use, you will need to obtain permission directly from the copyright holder. To view a copy of this license, visit http://creativecommons.org/ licenses/by/4.0/.
}

(C) The Author(s) 2019 